\title{
Quiet-Sun hydrogen Lyman- $\alpha$ line profile derived from SOHO/SUMER solar-disk observations *
}

\author{
S. Gunár ${ }^{1}$, P. Schwartz ${ }^{2}$, J. Koza ${ }^{2}$, and P. Heinzel ${ }^{1}$ \\ 1 Astronomical Institute, The Czech Academy of Sciences, 25165 Ondřejov, Czech Republic \\ e-mail: stanislav.gunar@asu.cas.cz \\ 2 Astronomical Institute of Slovak Academy of Sciences, 05960 Tatranská Lomnica, Slovak Republic \\ Received 5 September 2020 / Accepted 1 October 2020
}

\begin{abstract}
Context. The solar radiation in the Lyman- $\alpha$ spectral line of hydrogen plays a significant role in the illumination of chromospheric and coronal structures, such as prominences, spicules, chromospheric fibrils, cores of coronal mass ejections, and solar wind. Moreover, it is important for the investigation of the heliosphere, Earth's ionosphere, and the atmospheres of planets, moons, and comets.

Aims. We derive a reference quiet-Sun Lyman- $\alpha$ spectral profile that is representative of the Lyman- $\alpha$ radiation from the solar disk during a minimum of solar activity. This profile can serve as an incident radiation boundary condition for the radiative transfer modelling of chromospheric and coronal structures. Because the solar radiation in the Lyman lines is not constant over time but varies significantly with the solar cycle, we provide a method for the adaptation of the incident radiation Lyman line profiles (Lyman- $\alpha$ and higher lines) to a specific date. Moreover, we analyse how the change in the incident radiation influences the synthetic spectra produced by the radiative transfer modelling.

Methods. We used SOHO/SUMER Lyman- $\alpha$ raster scans obtained without the use of the attenuator in various quiet-Sun regions on the solar disk. The observations were performed on three consecutive days (June 24, 25, and 26, 2008) during a period of minimum solar activity. The reference Lyman- $\alpha$ profile was obtained as a spatial average over eight available raster scans. To take into account the Lyman- $\alpha$ variation with the solar cycle, we used the LISIRD composite Lyman- $\alpha$ index. To estimate the influence of the change in the incident radiation in the Lyman lines on the results of radiative transfer models, we used a 2D prominence fine structure model. Results. We present the reference quiet-Sun Lyman- $\alpha$ profile and a table of coefficients describing the variation of the Lyman lines with the solar cycle throughout the lifetime of SOHO. The analysis of the influence of the change in the incident radiation shows that the synthetic spectra are strongly affected by the modification of the incident radiation boundary condition. The most pronounced impact is on the central and integrated intensities of the Lyman lines. There, the change in the synthetic spectra can often have the same amplitude as the change in the incident radiation itself. The impact on the specific intensities in the peaks of reversed Lyman-line profiles is smaller but still significant. The hydrogen $\mathrm{H} \alpha$ line can also be considerably affected, despite the fact that the $\mathrm{H} \alpha$ radiation from the solar disk does not vary with the solar cycle.
\end{abstract}

Key words. Sun: UV radiation - Sun: filaments, prominences - Sun: atmosphere - methods: data analysis - techniques: spectroscopic

\section{Introduction}

The Lyman- $\alpha$ line of hydrogen (1215.67 $\AA$ ) is the most intense ultraviolet (UV) spectral line observed on the solar disk. As such, it plays a significant role in the illumination of chromospheric and coronal structures, such as prominences, spicules, chromospheric fibrils, solar wind, and cores of coronal mass ejections (CMEs). A well-determined Lyman- $\alpha$ spectrum of the solar disk is thus one of the crucial boundary conditions for the radiative transfer models of these structures. The Lyman- $\alpha$ illumination is often referred to as the Lyman- $\alpha$ incident radiation in the modelling context. Details on the radiative transfer modelling of the photosphere, chromosphere, and corona, are available in, for example, the review by Leenaarts (2020). Prominence modelling was reviewed by, for example, Gibson (2018) and Gunár (2014). An example of a recent study employing radiative transfer modelling in spicules is available in, for example, Tei et al. (2020), and an example of filament modelling is available in Schwartz et al. (2019). Information regarding the modelling of

* Data are only available at the CDS via anonymous $\mathrm{ftp}$ to cdsarc.u-strasbg.fr (130.79.128.5) or via http://cdsarc. u-strasbg.fr/viz-bin/cat/J/A+A/644/A109
Lyman- $\alpha$ in CME cores is available in Heinzel et al. (2016), and information regarding the modelling of Lyman- $\alpha$ in the solar wind is available in Dolei et al. (2019).

Both the solar Lyman- $\alpha$ line profile and the total Lyman$\alpha$ irradiance (i.e. the radiant flux received at a surface per unit area integrated over the wavelength range of Lyman- $\alpha$ ) are also important for the investigation of the Earth's ionosphere (see e.g. Raulin et al. 2013; Nina \& Čadež 2014), the heliosphere (see e.g. Bzowski et al. 2013; Gladstone et al. 2013; Koutroumpa et al. 2017), cometary atmospheres (see e.g. Pryor et al. 2013; Combi et al. 2019), the Moon (see e.g. Pryor et al. 2020; Retherford et al. 2020), Mars (see e.g. Bougher et al. 2017; Thiemann et al. 2017), and other planets. While the total Lyman- $\alpha$ irradiance is continuously monitored by a succession of instruments - SOLSTICE/UARS (Rottman \& Woods 1994), XPS/SORCE (Woods et al. 2005), and LYRA/PROBA2 (Dominique et al. 2013) - precise spectroscopic observations of the Lyman- $\alpha$ line on the solar disk are rare. This is because the Lyman- $\alpha$ line lies in the UV part of the spectrum, and, as such, it needs to be observed from above the Earth's atmosphere - and preferably above the geo-corona, which absorbs in the Lyman- $\alpha$ core. Moreover, its 
very high intensity and extreme UV wavelength range complicate the space-borne spectroscopic observations. Previously, calibrated solar disk Lyman- $\alpha$ (and Lyman- $\beta$ ) profiles obtained by the LPSP (Laboratoire de Physique Stellaire et Planétaire, Artzner et al. 1977; Bonnet et al. 1978) on board the OSO-8 (Orbiting Solar Observatory) were presented in Lemaire et al. (1978) and Gouttebroze et al. (1978). Solar disk Lyman- $\alpha$ observations were also obtained by Reeves (1976) using Skylab ATM (Apollo Telescope Mount) and by the HRTS (HighResolution Telescope and Spectrograph) rocket-flight instrument (Basri et al. 1979). Later, Lyman- $\alpha$ spectra of on-disk structures obtained using the Ultraviolet Spectrometer and Polarimeter (UVSP) instrument on board the SMM (Solar Maximum Mission) were analysed by Fontenla et al. (1988). High-resolution spectro-polarimetric Lyman- $\alpha$ observations of the solar disk were recently obtained by Kano et al. (2017) using the Chromospheric Lyman-Alpha Spectro-Polarimeter (CLASP) rocket experiment. All these observations were made within the geocorona and were thus affected by absorption in the Lyman- $\alpha$ line core. In the era of the SOHO (Solar and Heliospheric Observatory), which is located at the Lagrangian point L1 outside the influence of geo-corona, solar disk Lyman- $\alpha$ spectra were deduced by Lemaire et al. (1998) from the scattered light at the primary mirror of the SOHO/SUMER (Solar Ultraviolet Measurements of Emitted Radiation, Wilhelm et al. 1995) spectrograph. SOHO/SUMER typically observed solar-disk Lyman- $\alpha$ at a dedicated part of the detectors with an attenuating layer to protect them from the deterioration caused by the high Lyman- $\alpha$ intensity. This attenuator, however, was introduced non-linearity into the radiometric calibration, which makes the calibration of Lyman- $\alpha$ spectra difficult and unreliable. Only a small number of SOHO/SUMER Lyman- $\alpha$ observations without the use of the attenuator were carried out. The first such Lyman- $\alpha$ observations (obtained on June 24 and 25, 2008) with reduced incoming photon flux due to the partially closed aperture door of the telescope were presented and analysed by Curdt et al. (2008). Later, Tian et al. (2009a,b) analysed further SOHO/SUMER Lyman$\alpha$ observations of the quiet Sun and polar coronal holes. In the current work, we used the quiet-Sun Lyman- $\alpha$ observations that were obtained over three consecutive days between June 24 and 26,2008 . This set of eight SOHO/SUMER raster scans allows us to derive the reference quiet-Sun Lyman- $\alpha$ spectra unaffected by the geo-corona. In doing so, we complement the comprehensive study of the Lyman line series above the Lyman- $\alpha$ line carried out by Warren et al. (1998).

The Lyman- $\alpha$ radiation from the solar disk is not constant over time but varies significantly with the solar cycle. This variation is well demonstrated by, for example, the LISIRD composite Lyman- $\alpha$ index ${ }^{1}$ (Machol et al. 2019). In extreme cases, the values of this index exhibit differences between the minima and maxima of a cycle of up to $100 \%$. An empirical model of the solar Lyman- $\alpha$ irradiance variation was developed by Kretzschmar et al. (2018). This model is in a good agreement with the LISIRD composite Lyman- $\alpha$ index. Variations of the Lyman- $\alpha$ (and Lyman- $\beta$ ) spectra over solar cycle 23 were also studied by Lemaire et al. $(2005,2015)$. These authors used the technique of Lemaire et al. (1998) and observed the scattered light in SOHO/SUMER to derive full-disk Lyman- $\alpha$ profiles throughout cycle 23 . In the present study, we complement their work by using the SOHO/SUMER solar disk Lyman- $\alpha$ observations and the LISIRD composite Lyman- $\alpha$ index (Machol et al.

lasp.colorado.edu/lisird/data/composite_lyman_alpha
2019) to derive the variation of the Lyman- $\alpha$ spectra throughout cycle 24 and beyond.

In addition, in the present paper, we investigate the influence of the change in the illumination in the Lyman lines on the results of radiative transfer models. To do so, we used as an example the synthetic spectra produced by the $2 \mathrm{D}$ non-LTE (i.e. departures from local thermodynamic equilibrium) prominence vertical fine structure model from Heinzel \& Anzer (2001). These synthetic spectra, which are based on a realistic set of model input parameters (see Gunár et al. 2010), allowed us to estimate the degree by which the variation of the illumination during the solar cycle affects the Lyman lines as well as the $\mathrm{H} \alpha$ line. The paper is organized as follows. The SOHO/SUMER observations used for the derivation of the reference full-disk Lyman- $\alpha$ line profile are described in Sect. 2. The variation of the Lyman spectra with the solar cycle is addressed in Sect. 3. The influence of the illumination change on the synthetic spectra produced by prominence radiative transfer models is analysed in Sect. 4. In Sect. 5, we discuss the results, and we offer our conclusions in Sect. 6. Tables containing the reference Lyman- $\alpha$ profile can be found in Appendix A and as an electronic attachment. In Appendix B, we provide a table of coefficients describing the variation of the Lyman spectra throughout the lifetime of SOHO. These coefficients can be also downloaded as an electronic attachment.

\section{SOHO/SUMER observations}

We used a set of eight SOHO/SUMER Lyman- $\alpha$ raster scans of various quiet-Sun regions obtained with reduced incoming photon flux on June 24, 25, and 26, 2008. These observations correspond to the minimum of solar cycle 23. On June 24 and 25 , the rasters were pointed at the disk centre $(\mu=1.0)$, midway between the disk centre and the east limb $(\mu=0.85)$, as well as near the east limb ( $\mu$ from 0.47 to 0.0 ). On June 26 , pointing was at the disk centre and midway between the disk centre and the south pole $(\mu=0.85)$. More details are available in Table 1. In Fig. 1, we show the position of the rasters on the full-disk $\mathrm{H} \alpha$ images obtained by the Big Bear Solar Observatory and by the SMART telescope (Ueno et al. 2004) at the Hida Observatory. In Fig. 2, we plot the integrated intensity maps in each raster. All rasters have dimensions of $120 \times 120$ arcsec and consist of 80 slit positions with $\mathrm{N}-\mathrm{S}$ orientations. The slit has dimensions of $0.28 \times 120 \mathrm{arcsec}$. The exposure time is $15 \mathrm{~s}$, and the step between slit positions is 1.5 arcsec. The spectral data were recorded on the bare part of detector B (i.e. outside the attenuator) with the standard voltage of $5656 \mathrm{~V}$. The Lyman- $\alpha$ spectral profile was obtained separately in two halves and subsequently stitched together.

All Lyman- $\alpha$ observations used in this work were obtained with a partially closed aperture door of the telescope to decrease the incoming photon flux and thus protect the SOHO/SUMER detector. A detailed description of this observational technique is given in Curdt et al. (2008). The observed data were reduced and calibrated using standard SolarSoft procedures. The following procedures were applied in this order: decompression of binary data, dead-time correction, flat-fielding, local-gain correction, and correction for the geometrical distortion of the detector. Finally, the data were calibrated to radiometric units using the radiometry procedure. Technical details about the instrument, corrections, and calibration procedures are available in the SUMER Data Cookbook (Schühle 2003) and references therein. After the radiometric calibration, the obtained specific intensities were multiplied by a factor of five to compensate for the reduction of the observed signal due to the partially closed aperture 
Table 1. Used SOHO/SUMER Lyman- $\alpha$ raster scans.

\begin{tabular}{lccccc}
\hline \hline Raster position & Solar $X[\operatorname{arcsec}]$ & Solar $Y$ [arcsec] & $\mu$ interval & Date & Time [UT] \\
\hline Disk centre & 0 & 0 & $1.0 \pm 0.002$ & $2008 / 06 / 24$ & $18: 11: 32-18: 32: 44$ \\
Midway & -500 & 0 & $0.89-0.81$ & $2008 / 06 / 24$ & $18: 35: 23-18: 56: 37$ \\
Near limb & -900 & 0 & $0.47-0.00$ & $2008 / 06 / 24$ & $18: 58: 09-19: 19: 19$ \\
Disk centre & 0 & 0 & $1.0 \pm 0.002$ & $2008 / 06 / 25$ & $16: 00: 46-16: 21: 52$ \\
Midway & -500 & 0 & $0.89-0.81$ & $2008 / 06 / 25$ & $16: 23: 25-16: 44: 52$ \\
Near limb & -900 & 0 & $0.47-0.00$ & $2008 / 06 / 25$ & $16: 46: 24-17: 07: 32$ \\
Disk centre & 0 & 0 & $1.0 \pm 0.002$ & $2008 / 06 / 26$ & $21: 00: 44-21: 22: 26$ \\
Midway & 0 & -500 & $0.89-0.81$ & $2008 / 06 / 26$ & $21: 23: 59-21: 45: 25$ \\
\hline
\end{tabular}

Notes. The solar $X$ and $Y$ coordinates give the position of the centre of individual rasters.
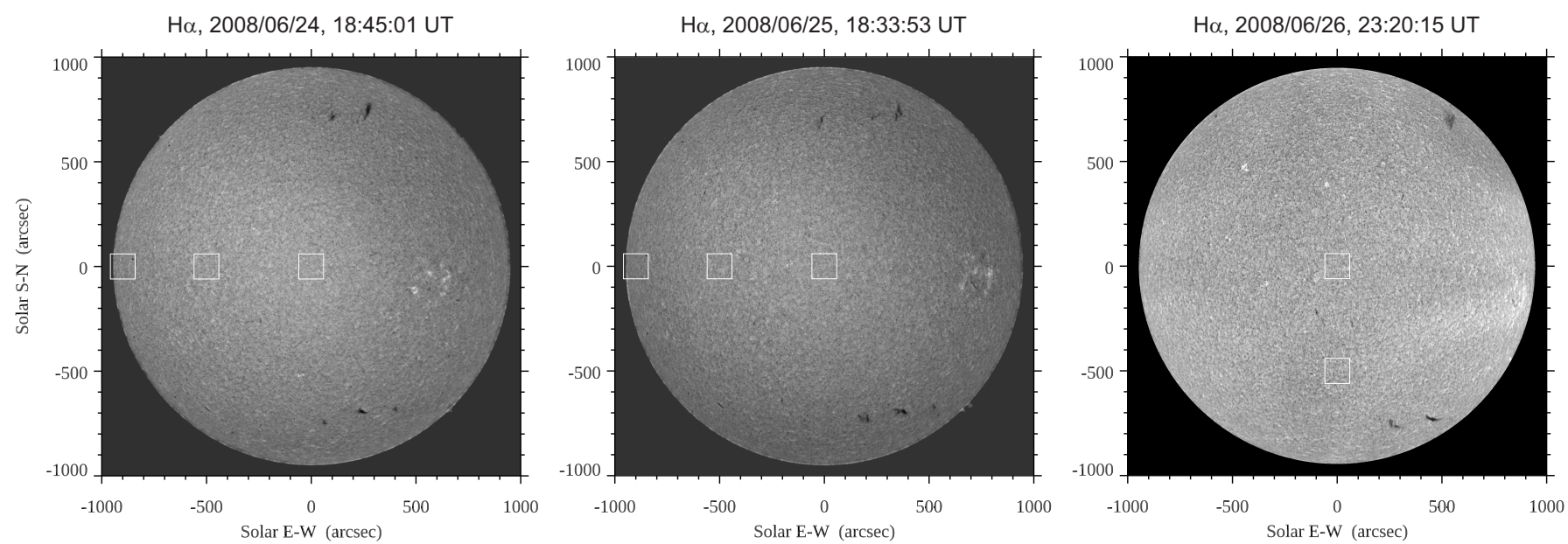

Fig. 1. Context $\mathrm{H} \alpha$ images obtained by the Big Bear Solar Observatory on June 24, 2008, at 18:45:01 UT (left) and on June 25, 2008, at 18:33:53 UT (middle), and by the SMART telescope at the Hida Observatory (right) on June 26, 2008, at 23:20:15 UT. Squares indicate the positions of the $\mathrm{SOHO} / \mathrm{SUMER}$ rasters used here. The contrast of the $\mathrm{H} \alpha$ images was enhanced, and the limb darkening was corrected.

door. The precision of the SOHO/SUMER absolute radiometric calibration was maintained to within $15 \%$ during the early years of operations (Wilhelm et al. 1997; Schühle et al. 1998); however, it may have decreased since. Due to the narrow width $(0.28 \mathrm{arcsec})$ of the slit used for the observations, the influence of the instrumental profile is small. We determined that the deconvolution of the instrumental profile modifies the observed Lyman- $\alpha$ intensities by up to $5 \%$. As this is below the overall uncertainties of the radiometric calibration, we used here the Lyman- $\alpha$ profiles as observed. Due to uncertainties in the SOHO/SUMER pointing precision, the solar $X$ and $Y$ coordinates referred to throughout this work may be shifted by up to \pm 15 arcsec. However, such shifts do not considerably affect our results.

\subsection{Analysis of the centre-to-limb variation}

To analyse the variation of the Lyman- $\alpha$ profiles across the solar disk, we selected seven regions in the rasters obtained on June 24 and 25 because the quiet-Sun limb observations were only performed on these days. In the selected regions (see Fig. 2), we averaged the specific Lyman- $\alpha$ intensity at each wavelength. We plot the averaged intensities for selected wavelengths in the lefthand column of Fig. 3. The regions centred at $\mu$ equal to 1.0, 0.85 , and 0.4 encompass large portions of the observed rasters. The averaging over such wide areas minimizes the influence of the significant local variations that are clearly demonstrated in all rasters and are shown in Fig. 2 by the presence of stochastically distributed bright and dark structures. Such a large local vari- ability of the Lyman- $\alpha$ spectra on the solar disk is caused by the significant differences between the network and inter-network regions. The effect of local intensity variations is apparent in the averaged intensities from the regions centred at $\mu$ equal to 0.3 , $0.25,0.2$, and 0.15 . These regions cover considerably smaller portions of the observed rasters and were selected to investigate the variation of the Lyman- $\alpha$ intensity close to the solar limb. The average intensities from these smaller regions vary considerably between neighbouring regions (see the left-hand column of Fig. 3). In the right-hand column of Fig. 3, we plot the averaged Lyman- $\alpha$ line profiles obtained from each region; we only plot half of the symmetrized Lyman- $\alpha$ profile. In general, the Lyman$\alpha$ profiles observed on the solar disk are typically asymmetric, with the dominant blue peak (see e.g. Curdt et al. (2008)). However, the variation of the intensities in the blue and red parts of the Lyman- $\alpha$ profile as a function of $\mu$ is very similar, with only a small difference $(\sim 10 \%)$ in some wavelengths. From Fig. 3 (right-hand column), it is clear that the profiles obtained at different regions vary. However, the available set of observed data is not broad enough for a statistically significant analysis of these variations.

To assess in more detail the centre-to-limb variation of the Lyman- $\alpha$ integrated intensity, we averaged the observed data obtained on all three days over concentric arcs with a width of 1 arcsec. The results (see Fig. 4) clearly show that the local variability of the integrated Lyman- $\alpha$ intensity is greater than any perceptible trend in the centre-to-limb variation. This is in agreement with the findings of Curdt et al. (2008). 

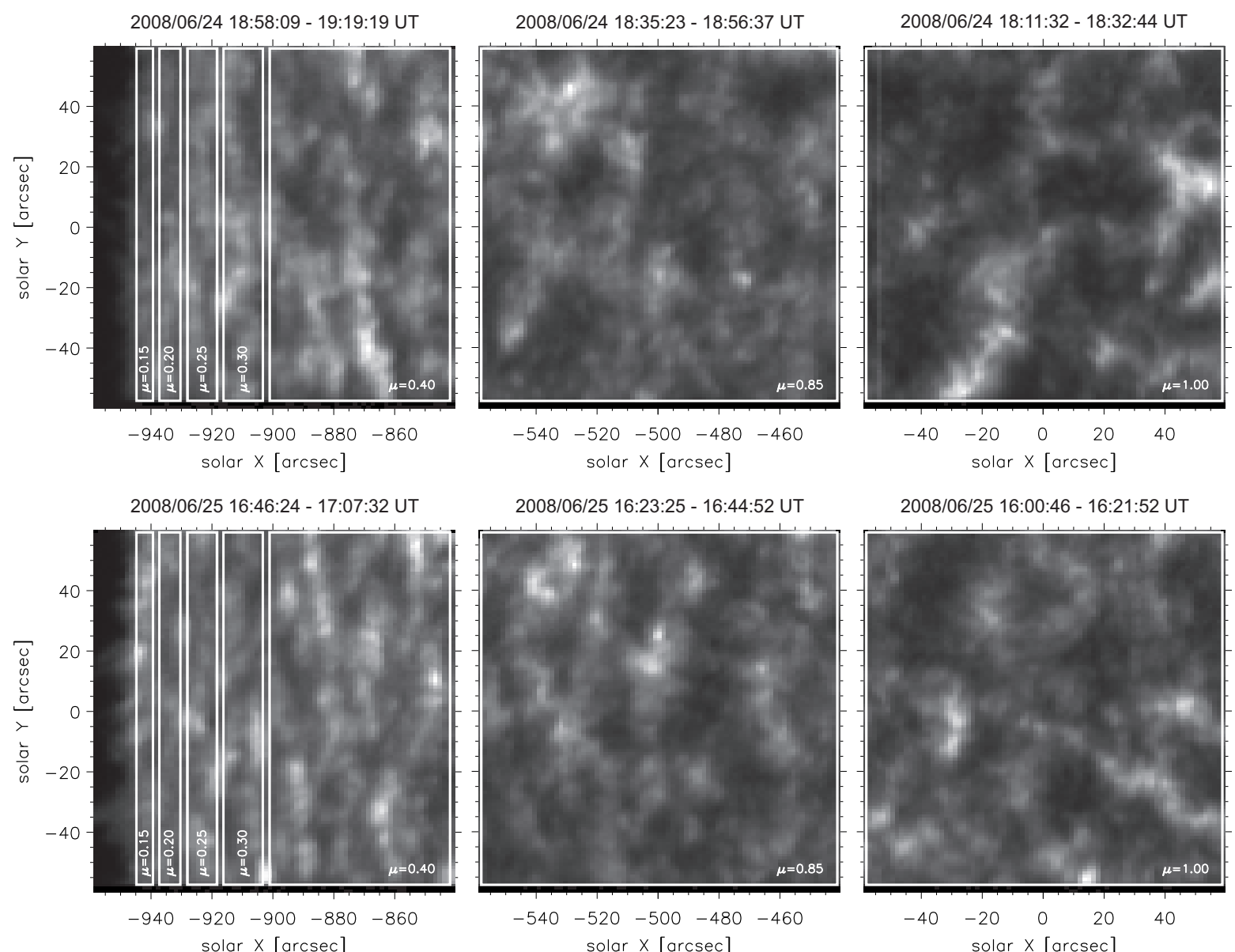

2008/06/25 16:00:46 - 16:21:52 UT
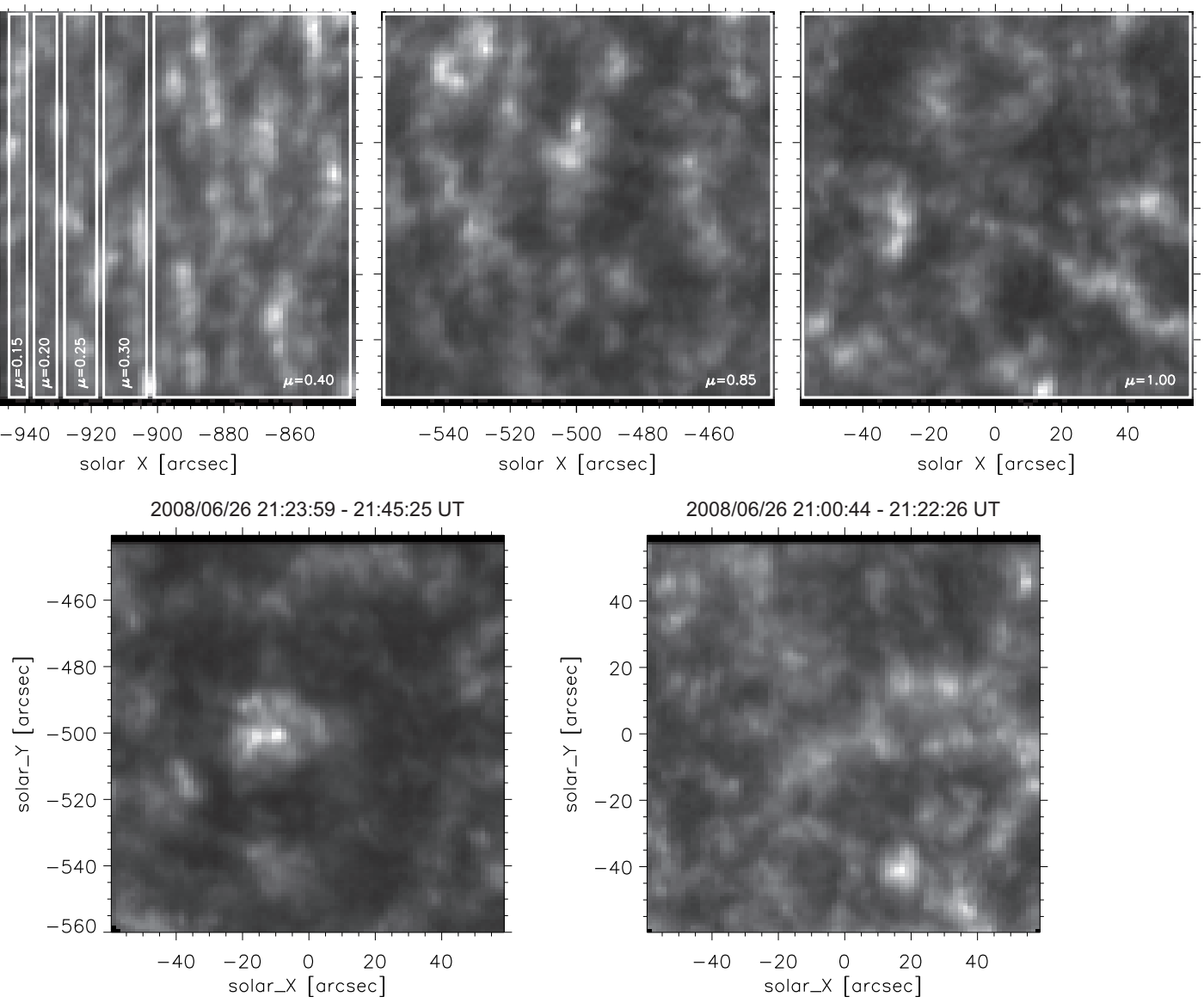

$-520-500-480$
solor $\times[$ orcsec $]$

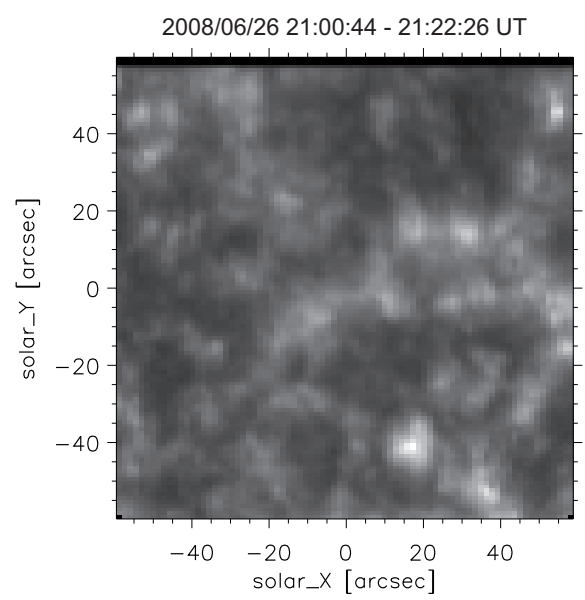

Fig. 2. Lyman- $\alpha$ integrated intensity maps in SOHO/SUMER rasters obtained on June 24, 2008 (top row), June 25, 2008 (middle row), and June 26, 2008 (bottom row). White rectangles in rasters in the upper and middle rows highlight the regions selected for spatial averaging. These regions correspond to Fig. 3. Each region is centred at the marked $\mu$ value.

\subsection{Full-disk Lyman- $\alpha$ profile}

Due to the absence of a clear centre-to-limb variation trend, we derived the full-disk Lyman- $\alpha$ profile by spatially averaging the specific intensities at each wavelength over all eight observed rasters. In this way, we obtained the reference quietSun Lyman- $\alpha$ profile that represents the minimum of solar activity. The integrated intensity of the reference Lyman- $\alpha$ profile is $7.274 \mathrm{erg} \mathrm{cm}^{-2} \mathrm{~s}^{-1} \mathrm{str}^{-1}$. We show the reference Lyman- $\alpha$ profile in the left-hand panel of Fig. 5, together with its uncertainties. In the estimation of the uncertainties, we took into account the findings of Wilhelm et al. (1997), who attribute the $\pm 15 \%$ uncertainty of SOHO/SUMER radiometric calibration to the dayto-day variation of the flat field. Thus, according to a general formula for error propagation, averaging over three days should lead to a reduction of the uncertainties from the admittedly optimistic $15 \%$ to $\pm(15 / \sqrt{3}) \%$. In the right-hand panel of Fig. 5, we plot one half of the symmetrized reference Lyman- $\alpha$ profile. In Appendix A, we provide the data of both the full and symmetrized reference Lyman- $\alpha$ profiles. The same data sets are available at the CDS. 
S. Gunár et al.: Quiet-Sun hydrogen Lyman- $\alpha$ line profile
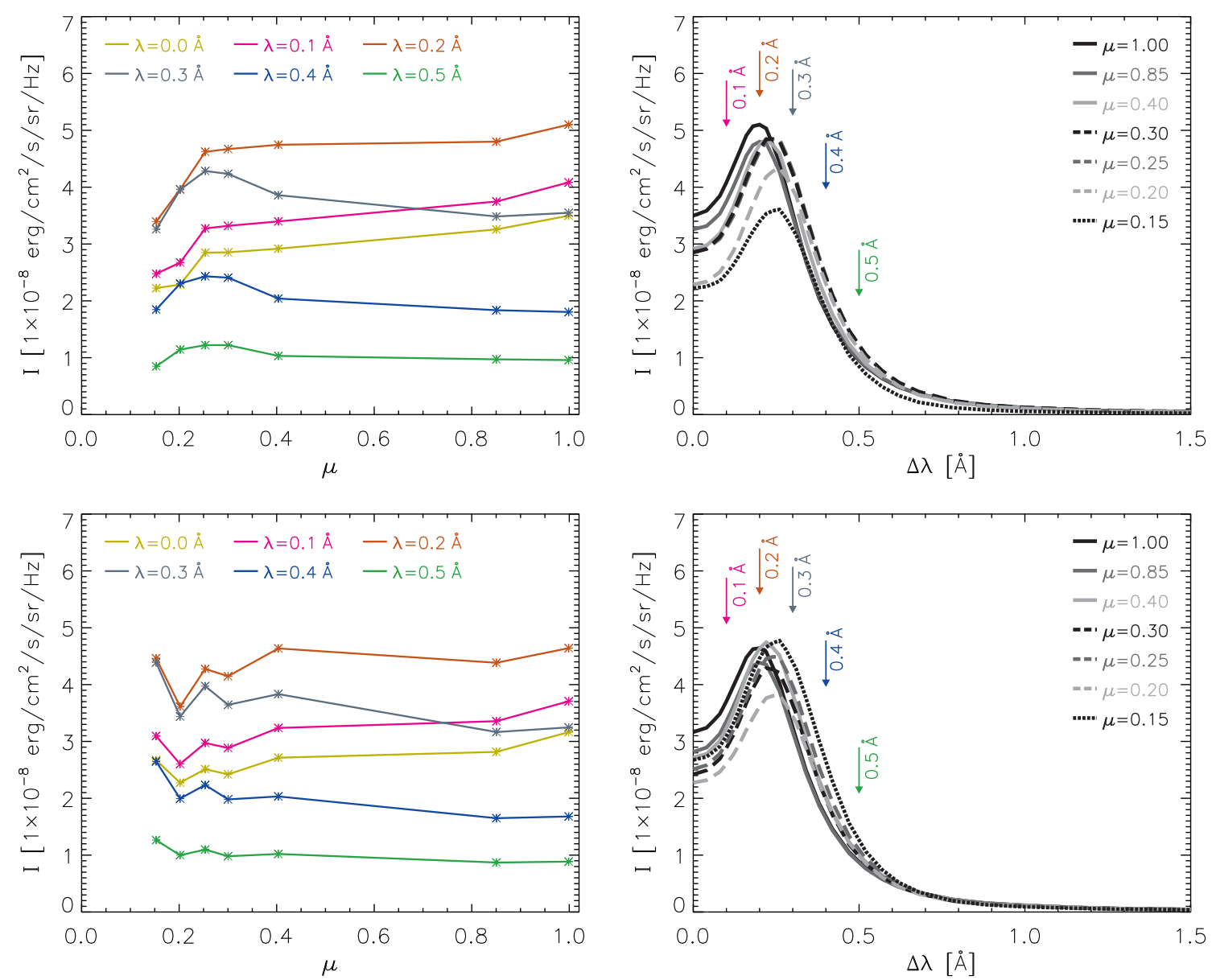

Fig. 3. Left column: spatially averaged Lyman- $\alpha$ intensities for selected wavelengths (marked in the upper left corner) as a function of $\mu$. Right column: spatially averaged Lyman- $\alpha$ symmetrized profiles obtained from regions corresponding to Fig. 2. Top row: data obtained on June 24,2008 and bottom row: data obtained on June 25, 2008.

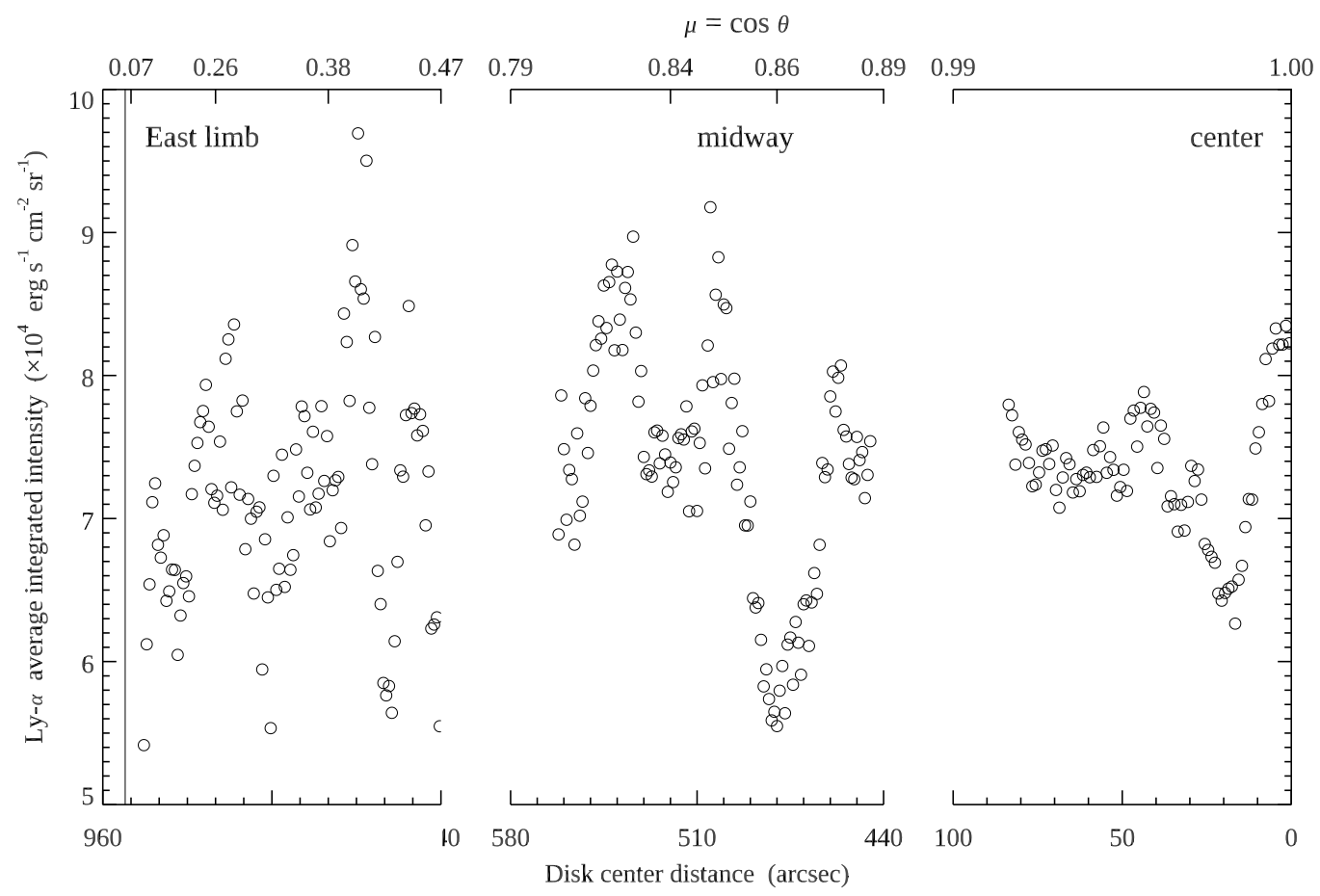

Fig. 4. Centre-to-limb variation of the Lyman- $\alpha$ wavelength-integrated intensities averaged over concentric arcs with a width of 1 arcsec. Each plotted circle in sections centre and midway represents an average from rasters obtained on June 24-26, 2008. For the near-limb section, only rasters from June 24 and 25 were used. The thin vertical line shows the solar radius of 952 arcsec as seen from SOHO. 

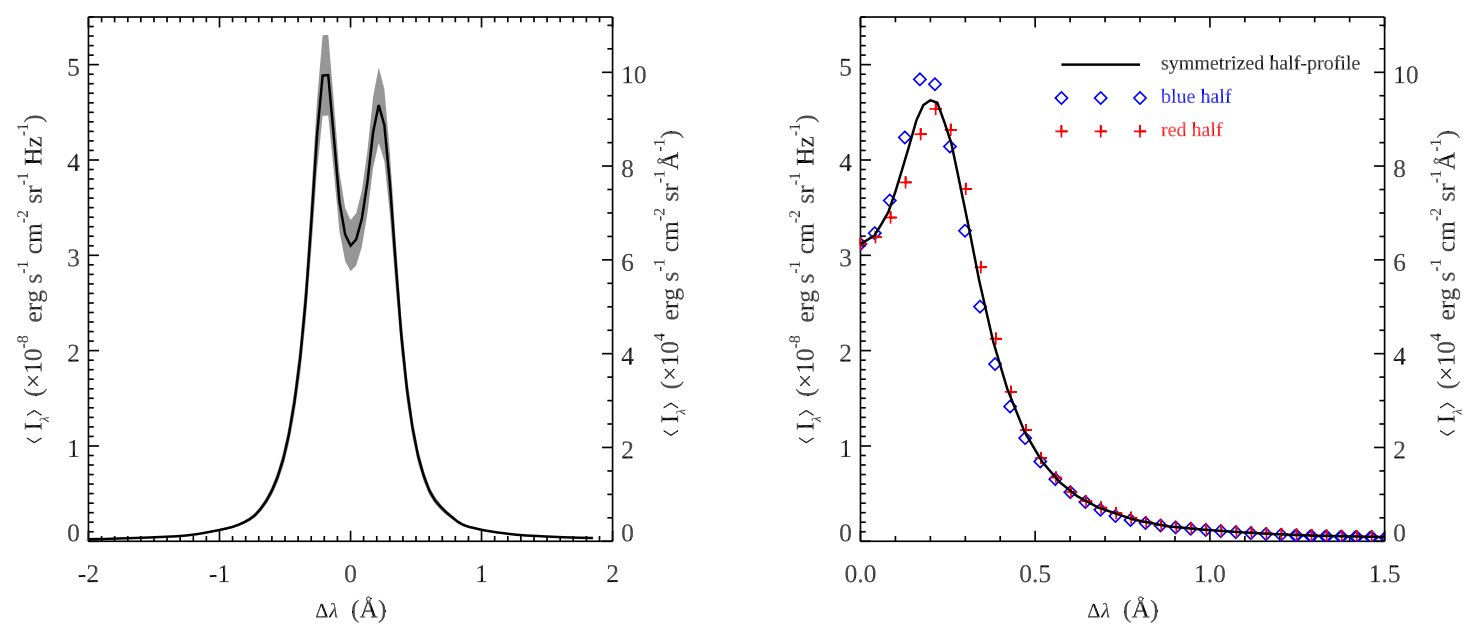

Fig. 5. Left panel: reference quiet-Sun Lyman- $\alpha$ profile obtained as an average over eight observed SOHO/SUMER rasters. The grey area indicates the uncertainty of the reference profile estimated to be $\pm(15 / \sqrt{3}) \%$. Right panel: half of the symmetrized reference Lyman- $\alpha$ profile with an indicated shape of the blue and the red part of the full profile.

\section{Variation with the solar cycle}

\subsection{Lyman- $\alpha$ line}

The Lyman- $\alpha$ radiation from the solar disk is not constant over time but varies significantly with the solar cycle. To take these changes into account, we used the LISIRD composite Lyman$\alpha$ index (Machol et al. 2019). The value of this index, shown in Fig. 6, clearly follows the solar cycle pattern and can vary considerably - extreme differences between the minima and maxima of a cycle can reach up to $100 \%$. The same index was also used by Lemaire et al. (2015) to investigate the variation of the Lyman- $\alpha$ spectra throughout solar cycle 23 .

In the present work, we used the LISIRD composite Lyman$\alpha$ index smoothed by the running mean over 400 days (13 months), which is usually used for the representation of solar cycle minima and maxima (see e.g. Hathaway 2015). The typical differences between the minima and maxima of the smoothed index (red line in Fig. 6) are around 50\%. We used the smoothed index to derive coefficients describing the change in the Lyman$\alpha$ illumination between the date when the reference Lyman$\alpha$ profile was obtained (June 25,2008 ) and the date when the observations analysed by the radiative transfer modelling were obtained. These coefficients can be derived for any date as a ratio between the value of the Lyman- $\alpha$ index on the day of observation and the value of the index on the reference day (June 25,2008 ), which is $0.605 \times 10^{-2} \mathrm{~W} \mathrm{~m}^{-2}$. In Appendix B, we list coefficients (obtained for the smoothed index) for selected days from the start of the SOHO observations until 2020. These coefficients are also available at the CDS. We note that we applied the same coefficients to the specific intensities throughout the Lyman- $\alpha$ profile. This is in agreement with the findings of Kretzschmar et al. (2018), whose empirical model shows a negligible wavelength dependence of the variation of Lyman- $\alpha$ irradiance with the solar cycle. We note, however, that the phenomenological model of the solar Lyman- $\alpha$ line from Kowalska-Leszczynska et al. (2018) points to a more pronounced wavelength dependence of the Lyman- $\alpha$ irradiance on the evolution of the solar activity.

\subsection{Higher Lyman lines}

While the Lyman- $\alpha$ line is the most intense source of illumination for the hydrogen radiative transfer modelling of chro- mospheric and coronal structures, the higher Lyman lines also play an important role. In the absence of a better source of information about their cyclic variations, we again used the LISIRD composite Lyman- $\alpha$ index as a proxy for the variation of higher Lyman lines. We note that the findings of Lemaire et al. (2012) show that the ratio of the irradiance in Lyman- $\alpha$ and Lyman$\beta$ lines is not constant over time. However, the differences in the ratio of these two lines appear to be considerably smaller than the variation of the irradiance over the solar cycle (see Lemaire et al. 2012 for more details). We thus assume here a constant ratio between Lyman- $\alpha$ and higher Lyman lines.

In the present work, we did not derive the solar disk spectra in the Lyman lines above Lyman- $\alpha$. We used the reference spectra provided by Warren et al. (1998). The data used by Warren et al. (1998) were also obtained by SOHO/SUMER on May 15, 1996, also within a period of minimum activity of solar cycle 22. However, there is a small difference (see Fig. 6) in the irradiance between the date used by Warren et al. (1998) and the date of the Lyman- $\alpha$ observations used in the present work (June $25,2008)$. Therefore, we first used a coefficient $(0.973)$ to normalize the intensity on these two dates, and then we applied the same coefficients as for the observations analysed in the present work. In Appendix B, we list the combined coefficients for the transformation of Warren et al. (1998) reference profiles to the selected dates.

\section{3. $\mathrm{H} \alpha$ line}

Another important source of illumination of chromospheric and coronal structures is the Balmer $\mathrm{H} \alpha$ line. However, the intensity of this line, in contrast to the lines of the Lyman series, does not change significantly with the solar cycle. For example, the MuSIL Corrected SORCE SIM index ${ }^{2}$ in the wavelength range centred at $6562 \AA$ shows very small differences (less than $1 \%$ ) between the minima and maxima of the $\mathrm{H} \alpha$ intensity. We can, therefore, assume the same $\mathrm{H} \alpha$ incident-radiation profile throughout the solar cycle. The reference profiles from David (1961) together with the disk continuum intensities from, for example, Allen (1976) are typically used for all Balmer lines.

\footnotetext{
2 lasp.colorado.edu/lisird/data/musil_sim
} 


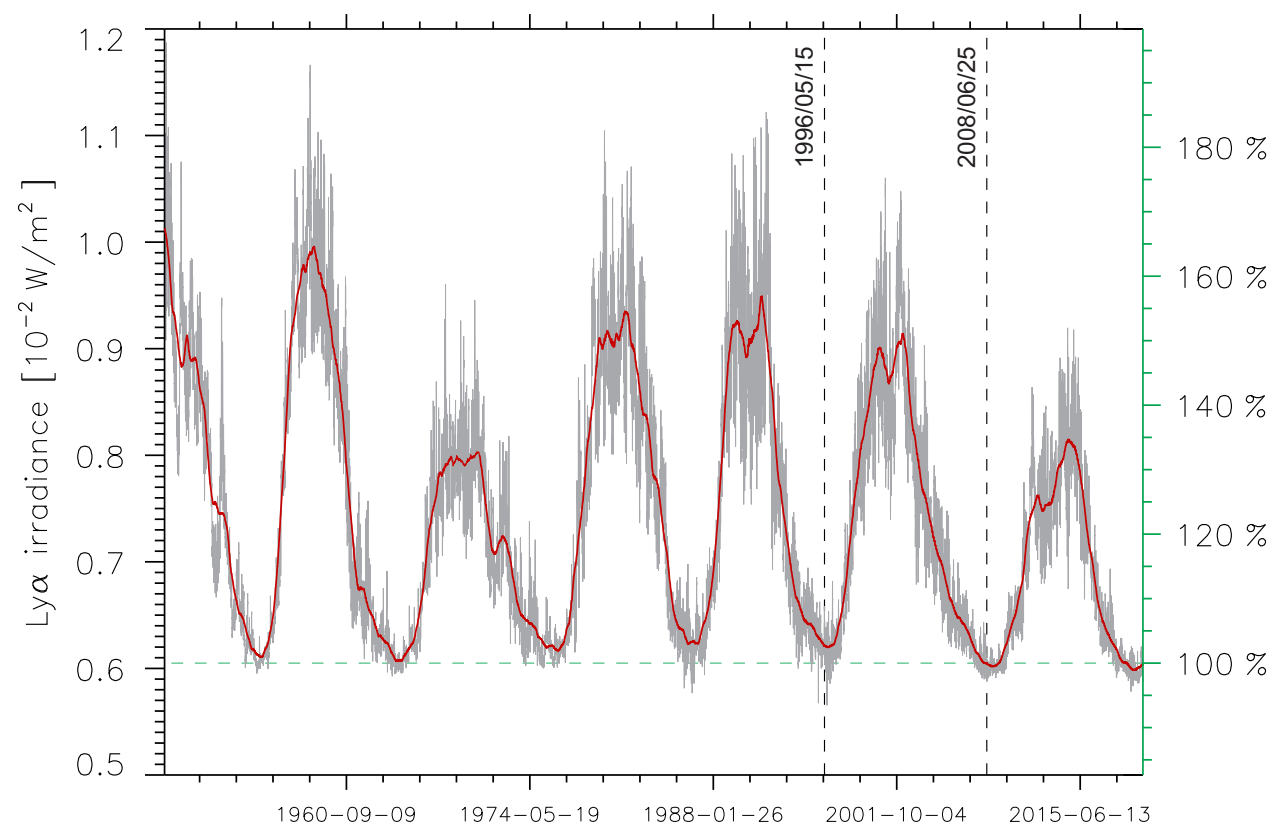

Fig. 6. Variation of the total Lyman- $\alpha$ irradiance based on the LISIRD composite Lyman- $\alpha$ index (grey line). The red line shows the same index smoothed by a running average over 400 days. Dashed vertical lines mark the reference date for the observations used in the current work (June 25, 2008) and the date (May $15,1996)$ of the observations used in Warren et al. (1998). The $y$-axis on the right-hand side gives the values of the index as a percentage of the value on the reference day $\left(0.605 \times 10^{-2} \mathrm{~W} \mathrm{~m}^{-2}\right)$, marked by the horizontal dashed line.

Table 2. Specific intensities in the peak of symmetrized Lyman- $\alpha$, Lyman- $\beta$, and Lyman- $\gamma$ incident-radiation profiles adapted to the selected dates that follow the change in illumination with the solar cycle.

\begin{tabular}{lcccc}
\hline \hline Date & $\begin{array}{c}\text { Lyman- } \alpha \text { peak intensity } \\
{\left[\mathrm{erg} \mathrm{cm}^{-2} \mathrm{~s}^{-1} \mathrm{str}^{-1} \mathrm{~Hz}^{-1}\right]}\end{array}$ & $\begin{array}{c}\text { Lyman- } \beta \text { peak intensity } \\
{\left[\mathrm{erg} \mathrm{cm}^{-2} \mathrm{~s}^{-1} \mathrm{str}^{-1} \mathrm{~Hz}^{-1}\right]}\end{array}$ & $\begin{array}{c}\text { Lyman- } \gamma \text { peak intensity } \\
{\left[\mathrm{erg} \mathrm{cm}^{-2} \mathrm{~s}^{-1} \mathrm{str}^{-1} \mathrm{~Hz}^{-1}\right]}\end{array}$ & $\begin{array}{c}\text { Difference } \\
\text { from } 2008 / 06 / 25\end{array}$ \\
\hline $2008 / 06 / 25$ & $4.74 \times 10^{-8}$ & $5.15 \times 10^{-10}$ & $1.23 \times 10^{-10}$ & $0 \%$ \\
$2010 / 06 / 24$ & $5.06 \times 10^{-8}$ & $5.51 \times 10^{-10}$ & $1.31 \times 10^{-10}$ & $7 \%$ \\
$2011 / 01 / 01$ & $5.28 \times 10^{-8}$ & $5.75 \times 10^{-10}$ & $1.37 \times 10^{-10}$ & $12 \%$ \\
$2011 / 06 / 24$ & $5.67 \times 10^{-8}$ & $6.16 \times 10^{-10}$ & $1.47 \times 10^{-10}$ & $20 \%$ \\
$2013 / 06 / 24$ & $5.99 \times 10^{-8}$ & $6.51 \times 10^{-10}$ & $1.55 \times 10^{-10}$ & $26 \%$ \\
$2014 / 06 / 24$ & $6.37 \times 10^{-8}$ & $6.93 \times 10^{-10}$ & $1.65 \times 10^{-10}$ & $34 \%$ \\
\hline
\end{tabular}

Notes. In the last column, we show relative differences between the intensities on the given date and the reference date June $25,2008$.

\section{Influence of the incident-radiation change on synthetic profiles}

In the previous section, we discussed the fact that the solar radiation in Lyman lines is not constant over time but changes significantly with the solar cycle. We have also presented a method that can be used to adapt the reference incident radiation profiles to a specific date when a set of observations analysed by radiative transfer modelling was obtained. Such a method can lead to an increased accuracy of the boundary conditions of radiative transfer models.

To estimate the extent of the influence of the illumination changes on the results of radiative transfer models, we used the 2D non-LTE model of vertical prominence fine structures developed by Heinzel \& Anzer (2001). This model was used for the analysis of prominence spectral observations by, for example, Gunár et al. (2010, 2012, 2014). In the present work, we adopted a set of model input parameters derived as a good fit to observations of a quiescent prominence by Gunár et al. (2010), referred to as MODEL1 therein and hereafter. This allowed us to analyse the influence of the modified incident radiation intensities on a model with a realistic set of input parameters (for more details on the parameters of the model, see Table 2 of Gunár et al. 2010). We note that the analysis presented here serves only as an estimate of the influence of varying illumination on the results of radiative transfer models. A more in-depth investigation involving models of different solar structures would be needed to reveal the true extent of this influence. However, such an investigation is beyond the scope of the current paper.

To follow the cyclic change in the solar disk radiation in Lyman lines, we selected five ad hoc dates representing the increase in the Lyman- $\alpha$ intensity from the minimum of the cycle to its maximum. The specific intensities in the peak of symmetrized Lyman- $\alpha$, Lyman- $\beta$, and Lyman- $\gamma$ incident radiation profiles for these dates are listed in Table 2. The reference date is the day of observation used in the present work (June 25, 2008), which represents a minimum of the solar activity. For each date, we computed MODEL1 in the single-thread configuration and produced the synthetic spectra of the Lyman lines and the $\mathrm{H} \alpha$ line. The 2D prominence fine structure model used here produces synthetic line profiles with a variety of shapes and intensities, depending on the orientation of the line-of-sight and its position along the length (or width) of the model. More details are available in, for example, Sect. 5 of Heinzel et al. (2005) or Sect. 4 of Gunár et al. (2007). In the present work, we took into account the synthetic profiles obtained with the line-of-sight perpendicular to the longer dimension of the model, that is to say, perpendicular to the magnetic field. Only the profiles with intensities higher than $20 \%$ of the maximum intensity in each spectral line are used. For consistency with observations, the resulting 

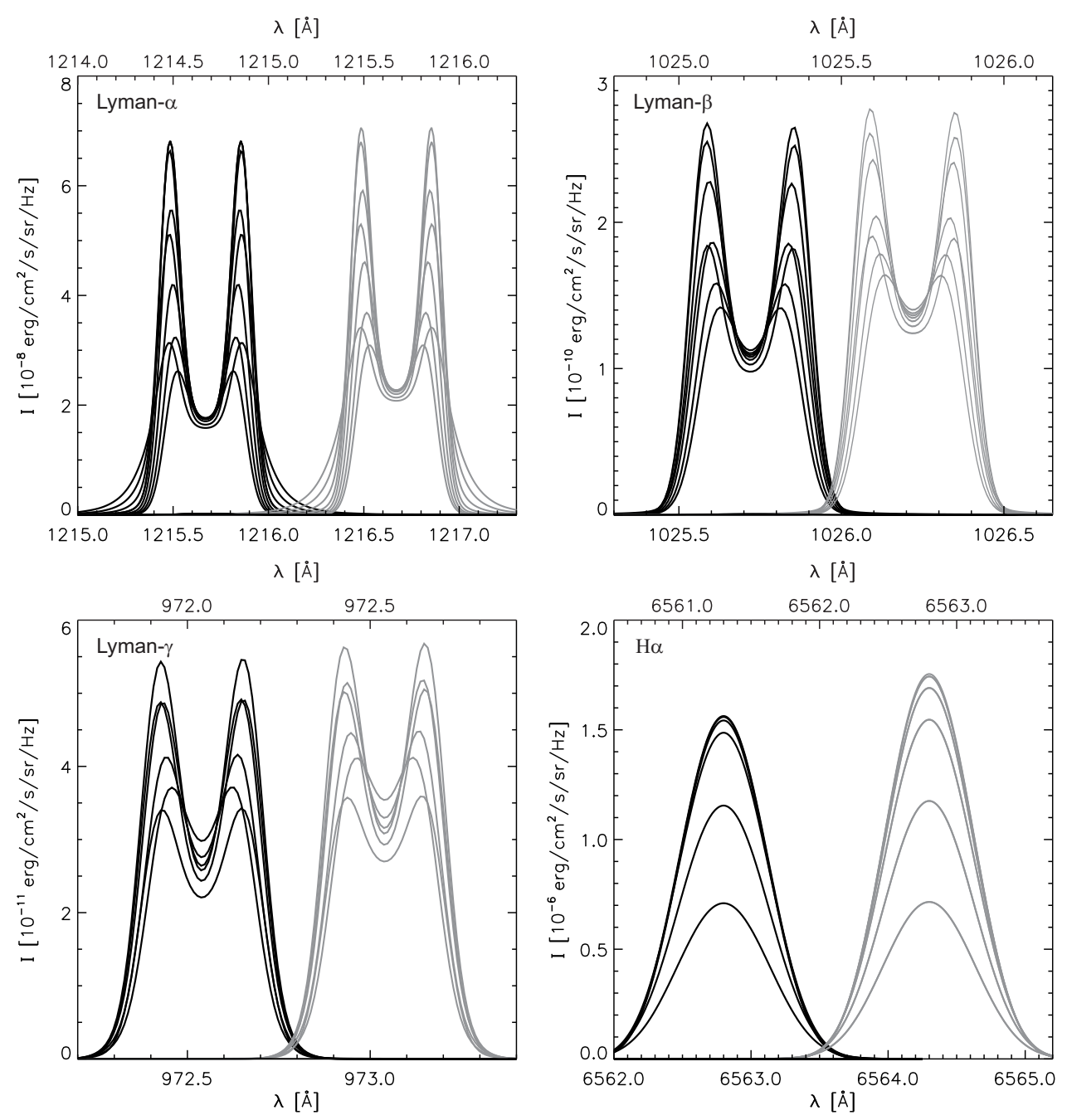

Fig. 7. Comparison of synthetic spectra produced by MODEL1 in the singlethread configuration with the illumination data for the reference date June 25, 2008 (black lines) and the date with the maximum illumination change June 24, 2014 (grey lines). We plot only the reversed profiles obtained at different positions along the length of the used $2 \mathrm{D}$ prominence fine structure model. We note that the bottom $x$-axis in each panel corresponds to the black profiles and the top $x$-axis to the grey profiles. All plotted profiles are convolved with the instrumental profiles of SOHO/SUMER (Lyman lines) or Meudon/MSDP $(\mathrm{H} \alpha)$.

synthetic spectra were convolved with the instrumental profile of SOHO/SUMER (see e.g. Sect. 3.1 of Gunár et al. 2008) and of the Meudon/MSDP (Multichannel Subtractive Double Pass) spectrograph working at the Meudon Solar Tower (Mein 1991).

We used three characteristics of the synthetic profiles for the analysis of the effect of the illumination change. These are the specific intensity in the centre of the line, its integrated intensity, and the maximum specific intensity in the peaks of the reversed profiles. We took into account only reversed profiles with peak intensities of more than $120 \%$ of the corresponding line-centre intensity. We plot these reversed profiles in Fig. 7 to illustrate the changes in the synthetic profiles due to modified incident radiation intensities. In this figure, we compare the synthetic profiles for the reference date June 25, 2008 (black lines) and the profiles for June 24, 2014 (grey lines). In Table 3, we list the relative differences between the central, integrated, and peak intensities obtained for the selected dates and those obtained for the reference date. For each date, spectral line, and analysed profile characteristic, we list the maximum, median, and minimum differences. We note that we do not list the peak intensities for the $\mathrm{H} \alpha$ line because the obtained synthetic profiles are not reversed.

Table 3 clearly shows that the centre of the Lyman- $\alpha$ line is strongly affected by the change in the incident radiation. In fact, the relative differences between the synthetic spectra obtained with the incident radiation data for June 24, 2014, and those obtained for the reference date are practically the same, as is the amplitude of change of the incident radiation itself. Moreover, these differences vary only slightly between the maximum and minimum values (see the second column of Table 3 ). The maximum differences in the centre of Lyman- $\beta$ and Lyman- $\gamma$ lines are similar to the differences in the centre of Lyman- $\alpha$. However, the variation between the maximum and minimum differences is more pronounced in these lines. The maximum relative differences between the integrated intensities of all three Lyman lines are also comparable to the amplitude of change in the incident radiation. The modification of the incident radiation has the least impact on the specific intensity in the peaks of the reversed profiles of Lyman lines. However, even here the peak intensities may change by up to two-thirds of the amplitude of change of the incident radiation. Only the most intense peaks are practically unaffected by the change in the incident radiation. This contrast between the influence of the modified illumination on the centre and the peaks of synthetic profiles can be seen in Fig. 7. Interestingly, the central and integrated intensities of the $\mathrm{H} \alpha$ line can also be significantly affected by the change in illumination in the Lyman lines. This is despite the fact that the illumination in the $\mathrm{H} \alpha$ line itself is constant (see Sect. 3.3). Both the specific intensity in the $\mathrm{H} \alpha$ line centre and its integrated intensity are typically affected by around one-fifth of the amplitude of change of the incident radiation in Lyman lines. However, this influence can increase to up to two-fifths in the most intense $\mathrm{H} \alpha$ profiles.

We also performed a further assessment of the influence of changing illumination on the modelling results, this time using the multi-thread configurations of MODEL1 with randomly 
Table 3. Relative differences between the central, integrated, and peak intensities of synthetic profiles obtained by MoDEL1 for the selected dates and the intensities obtained for the reference date (June 25, 2008).

\begin{tabular}{|c|c|c|c|c|c|c|c|c|c|c|c|c|}
\hline \multirow{2}{*}{ Date } & \multicolumn{3}{|c|}{ Lyman- $\alpha$ intensity } & \multicolumn{3}{|c|}{ Lyman $\beta$ intensity } & \multicolumn{3}{|c|}{ Lyman- $\gamma$ intensity } & \multicolumn{2}{|c|}{$\mathrm{H} \alpha$ intensity } & \\
\hline & Centre & Integral & Peak & Centre & Integral & Peak & Centre & Integral & Peak & Centre & Integral & \\
\hline \multirow{3}{*}{$2010 / 06 / 24$} & $7 \%$ & $6 \%$ & $5 \%$ & $6 \%$ & $6 \%$ & $3 \%$ & $6 \%$ & $6 \%$ & $2 \%$ & $3 \%$ & $3 \%$ & $\operatorname{Max}$ \\
\hline & $6 \%$ & $5 \%$ & $2 \%$ & $5 \%$ & $3 \%$ & $1 \%$ & $3 \%$ & $2 \%$ & $1 \%$ & $1 \%$ & $1 \%$ & Median \\
\hline & $6 \%$ & $2 \%$ & - & $2 \%$ & $1 \%$ & - & $1 \%$ & $1 \%$ & - & - & - & Min \\
\hline \multirow{3}{*}{ 2011/01/01 } & $11 \%$ & $11 \%$ & $7 \%$ & $10 \%$ & $10 \%$ & $5 \%$ & $10 \%$ & $9 \%$ & $4 \%$ & $5 \%$ & $5 \%$ & $\operatorname{Max}$ \\
\hline & $10 \%$ & $9 \%$ & $3 \%$ & $8 \%$ & $5 \%$ & $2 \%$ & $5 \%$ & $3 \%$ & $2 \%$ & $2 \%$ & $2 \%$ & Median \\
\hline & $10 \%$ & $3 \%$ & $1 \%$ & $3 \%$ & $2 \%$ & $1 \%$ & $1 \%$ & $1 \%$ & $1 \%$ & - & - & Min \\
\hline \multirow{3}{*}{$2011 / 06 / 24$} & $19 \%$ & $18 \%$ & $13 \%$ & $18 \%$ & $17 \%$ & $9 \%$ & $16 \%$ & $16 \%$ & $6 \%$ & $8 \%$ & $9 \%$ & Max \\
\hline & $17 \%$ & $15 \%$ & $5 \%$ & $14 \%$ & $8 \%$ & $4 \%$ & $9 \%$ & $5 \%$ & $3 \%$ & $4 \%$ & $3 \%$ & Median \\
\hline & $16 \%$ & $5 \%$ & $1 \%$ & $6 \%$ & $4 \%$ & $1 \%$ & $2 \%$ & $2 \%$ & $2 \%$ & - & - & Min \\
\hline \multirow{3}{*}{ 2013/06/24 } & $25 \%$ & $24 \%$ & $17 \%$ & $24 \%$ & $22 \%$ & $13 \%$ & $22 \%$ & $21 \%$ & $9 \%$ & $11 \%$ & $11 \%$ & Max \\
\hline & $23 \%$ & $20 \%$ & $7 \%$ & $19 \%$ & $11 \%$ & $5 \%$ & $12 \%$ & $7 \%$ & $4 \%$ & $5 \%$ & $5 \%$ & Median \\
\hline & $22 \%$ & $6 \%$ & $2 \%$ & $8 \%$ & $5 \%$ & $2 \%$ & $3 \%$ & $3 \%$ & $3 \%$ & $1 \%$ & - & Min \\
\hline \multirow{3}{*}{ 2014/06/24 } & $33 \%$ & $32 \%$ & $22 \%$ & $31 \%$ & $29 \%$ & $16 \%$ & $29 \%$ & $28 \%$ & $11 \%$ & $14 \%$ & $15 \%$ & Max \\
\hline & $30 \%$ & $26 \%$ & $10 \%$ & $25 \%$ & $15 \%$ & $7 \%$ & $16 \%$ & $9 \%$ & $6 \%$ & $6 \%$ & $6 \%$ & Median \\
\hline & $29 \%$ & $8 \%$ & $3 \%$ & $11 \%$ & $7 \%$ & $2 \%$ & $4 \%$ & $3 \%$ & $3 \%$ & $1 \%$ & - & Min \\
\hline
\end{tabular}

Notes. For each spectral line and analysed profile characteristic, we list the maximum, median, and minimum differences.

assigned line-of-sight velocities (see Gunár et al. 2010, for more details). When we used a multi-thread model with ten identical threads (each with the input parameters of MoDEL1) and the line-of-sight velocities selected randomly from an interval $\pm 10 \mathrm{~km} \mathrm{~s}^{-1}$, we found that, depending on the actual setup of lineof-sight velocities and the positional shifts in the multi-thread model, the synthetic profiles were affected to a slightly lesser degree than those produced by the single-thread model.

\section{Discussion}

The integrated intensity of the reference quiet-Sun Lyman- $\alpha$ profile from Fig. $5\left(7.36 \times 10^{4} \mathrm{erg} \mathrm{cm}^{-2} \mathrm{~s}^{-1} \mathrm{str}^{-1}\right)$ translates to a total Lyman- $\alpha$ irradiance of $0.501 \times 10^{-2} \mathrm{~W} \mathrm{~m}^{-2}$. However, this value is about $20 \%$ lower than the value of the LISIRD composite Lyman- $\alpha$ index on the reference date (June 25, 2008), which is $0.605 \times 10^{-2} \mathrm{~W} \mathrm{~m}^{-2}$. This discrepancy can be caused by a combination of various factors. The first is the uncertainty in the calibration of the SOHO/SUMER data used here, which is $\pm(15 / \sqrt{3}) \%$, together with the uncertainty of the LISIRD composite Lyman- $\alpha$ index calibration. The estimated uncertainty of the value of the index on the reference date is around 6\% (see Machol et al. 2019, for more details). The second factor is the presence of an active region and coronal holes at each pole during the dates when SOHO/SUMER observations were obtained (see Fig. 1). Both these features affect (and probably increase) the total Lyman- $\alpha$ irradiance represented by the LISIRD composite Lyman- $\alpha$ index, but they do not affect the averaged quiet-Sun reference profile derived from the SOHO/SUMER raster scans. To quantify the extent of the influence of active regions, plages, and coronal holes on the Lyman- $\alpha$ irradiance, we will need to analyse detailed Lyman- $\alpha$ spectral observations of these features. However, such an analysis is beyond the scope of the current paper.

For the assessment of the influence of the varying solar illumination on the synthetic spectra, we used the LISIRD com- posite Lyman- $\alpha$ index smoothed by a running averaging over 400 days (see Sect. 3.1). However, from Fig. 6, it is clear that the variations in the smoothed index are considerably smaller than the variations in the actual, daily-averaged values of the index itself. As we demonstrated in the previous section, the change in incident radiation can have a significant effect on the synthetic spectra. Therefore, for applications where it is necessary to employ the most accurate incident radiation data, we would suggest using the actual values of the composite Lyman- $\alpha$ index or other shorter-term averaging. We note, however, that the use of a running mean over a period other than 400 days will result in coefficients that vary from those listed in Appendix B. In the case when the actual, daily-averaged values of the Lyman- $\alpha$ index are used, it is important to take into account the fact that these values correspond to the radiation from the solar disk as seen from Earth. Therefore, the actual values should be used for the modelling of on-disk chromospheric or coronal structures that are not too distant from the disk centre. On the other hand, the limb structures, such as prominences, spicules, or CME cores, are illuminated only by a portion of the solar disk visible from the Earth and by a portion of the disk beyond the limb. However, the radiation from beyond the limb is obviously not included in the actual values of the LISIRD composite Lyman- $\alpha$ index. Therefore, special care should be exercised when these values are used for the analysis of the limb observations.

Realistic determination of the Lyman- $\alpha$ incident radiation profile is critically important for the diagnostics of the physical properties of the chromospheric and coronal structures with prominence-like conditions. This is because the Lyman- $\alpha$ line profiles emerging from these structures are produced by the combined effect of their physical conditions, the illumination from the solar surface, and the properties of the spectral-line formation mechanism in the regime of partial redistribution (PRD). For isothermal-isobaric prominence slab models, Heinzel et al. (1987) demonstrated that the emergent Lyman- $\alpha$ profile 
substantially differs when modelled with realistic PRD or with approximate complete redistribution (CRD). This is because, at low prominence densities, the photon scattering plays a dominant role in the line formation. This scattering is relatively well described by CRD only in the Lyman- $\alpha$ line core up to approximately three Doppler widths, while PRD is needed in the wings. Under typical prominence temperatures, three Doppler widths amount to about $0.15 \AA$, while the peaks of the Lyman- $\alpha$ incident radiation profile are located around $0.2 \AA$ from the line centre (see Fig. 5). This means that the peaks of the incident radiation profile are partially reproduced by quasi-coherent scattering (which is not the case of CRD). As a result, the line-core intensity is almost the same for both PRD and CRD, but the peaks are produced predominantly in the PRD regime. Moreover, far wings are much lower in the PRD case compared to CRD, which is a well-known effect in stellar atmospheres. Furthermore, it is interesting to note that the Lyman- $\alpha$ line-centre intensity in the 1D models is practically equal to the incident line-centre intensity multiplied by the geometrical dilution factor (see Heinzel et al. 1987). This is also true in the 2D prominence fine structure model used here. The aspects of the Lyman- $\alpha$ line formation mechanism described above have several consequences related to the shape and intensity of the incident radiation profile. First, the peaks of the synthetic Lyman- $\alpha$ profiles depend on the peaks of the incident radiation profile because they are quasi-coherently reproduced. However, the degree of the reproduction depends mainly on the electron density, which is a key parameter for the determination of departures from CRD. At high densities, the line formation mechanism in the peaks would approach the CRD regime. However, under the prominence-like conditions, the electron density is relatively low and the formation mechanism is in the PRD regime. Second, the synthetic intensity in the Lyman- $\alpha$ line core depends on the temperature and density structure of the prominence-corona transition region (PCTR), where, in the case of prominence-like structures, Lyman- $\alpha$ is formed due to its very high optical thickness. The dependence is caused by the thermal component of the Lyman- $\alpha$ line source function, which becomes important at higher temperatures. Therefore, any unrealistic determination of the line-core intensity of the Lyman- $\alpha$ incident radiation profile significantly decreases the accuracy of the plasma temperature diagnostics. We note that similar effects also take place in the case of the Lyman- $\beta$ line, but the formation of this line is less sensitive to PRD due to the strong coupling with the $\mathrm{H} \alpha$ line, which is formed in the CRD regime.

The Lyman- $\alpha$ profile observed on the solar disk is in general asymmetric, with a stronger blue peak, as was shown by Curdt et al. (2008) and Tian et al. (2009a,b). These authors also showed that the Lyman- $\beta$ line profile obtained on the solar disk generally shows an opposite asymmetry, with a stronger red peak. This behaviour might be caused by differential flows in the solar atmosphere due to a mechanism similar to that demonstrated by Gunár et al. (2008) in prominences. However, detailed causes of these opposing asymmetries of Lyman- $\alpha$ and higher Lyman lines are not yet clearly understood. The reference full-disk Lyman- $\alpha$ profile presented here exhibits the same stronger-blue-peak asymmetry. Such an asymmetry might play a negligible role in a majority of radiative transfer models. Indeed, many of the current models assume symmetric incident radiation profiles - the reason for which we also provide here the symmetrized reference Lyman- $\alpha$ profile (Fig. 5). However, the true influence of the asymmetries of the incident radiation profiles will need to be investigated in the future in greater detail. On the other hand, it is important to note that asymmetries in the incident radiation profiles may play a more significant role in the radiative transfer of polarized light, especially when the $3 \mathrm{D}$ polarized light radiative transfer is considered (see e.g. the 3D polarized radiative transfer code PORTA by Štěpán \& Trujillo Bueno 2013). This is because the polarization is sensitive to the breaking of symmetries. However, the results of polarized light radiative transfer modelling might be even more sensitive to the local inhomogeneities of the illumination from the solar disk than to the asymmetries of the incident radiation profiles.

\section{Conclusions}

We derived the reference quiet-Sun Lyman- $\alpha$ spectral profile, which represents the Lyman- $\alpha$ radiation from the solar disk during the minimum of the solar activity. This profile (Fig. 5) can serve as a representative illumination (incident radiation) boundary condition for radiative transfer models of chromospheric and coronal structures, such as prominences, spicules, chromospheric fibrils, solar wind, and CME cores. Moreover, solar Lyman- $\alpha$ profile and the total Lyman- $\alpha$ irradiance are important for the Earth's ionosphere, the heliosphere, and the atmospheres of planets, moons, and comets.

To derive the reference Lyman- $\alpha$ profile, we used what is, to our knowledge, the most comprehensive data set of SOHO/SUMER Lyman- $\alpha$ observations of the solar disk obtained without the use of the attenuator. This data set comprises eight Lyman- $\alpha$ raster scans obtained in various quiet-Sun regions over three consecutive days (June 24-26, 2008) with a reduced incoming photon flux due to a partially closed aperture door of the telescope. The rasters obtained on June 24 and 25 were previously presented and analysed by Curdt et al. (2008). We provide the reference Lyman- $\alpha$ profile in a tabular form in Appendix A and at the CDS. As we discuss in Sect. 2.2, the uncertainties in the determination of the reference profile are $\pm(15 / \sqrt{3}) \%$. These are caused by the uncertainty of $\pm 15 \%$ (at best) in the SOHO/SUMER radiometric calibration (Wilhelm et al. 1997; Schühle et al. 1998) alleviated by the use of observations obtained on three days.

Solar radiation in Lyman lines is not constant over time but varies significantly (up to $100 \%$ ) with the solar cycle (see Sect. 3). Such a dramatic change in the boundary conditions of radiative transfer models can have a profound effect on their results, as we show in this work. To take the variation of Lyman$\alpha$ with the solar cycle into account, we present in Sect. 3.1 a method for adapting the incident radiation Lyman line profiles to a specific date. This method uses the LISIRD composite Lyman$\alpha$ index (Machol et al. 2019) to derive coefficients for the modification of the reference Lyman- $\alpha$ profile presented here and the reference spectra of higher Lyman lines from Warren et al. (1998). The list of coefficients provided in Appendix B (and at the CDS) covers the lifetime of SOHO.

In the present work, we used the $2 \mathrm{D}$ non-LTE radiative transfer model of prominence vertical fine structures (Heinzel \& Anzer 2001) to analyse the influence of the change in illumination in the Lyman lines on the synthetic spectra. We used this model as an example that allowed us to estimate the impact of the illumination change on synthetic spectra, which are in broad agreement with prominence observations (see Gunár et al. 2010, for more details). However, a more thorough investigation employing models of various chromospheric and coronal structures will be needed to establish the true extent of the influence of varying incident radiation boundary conditions on the 
modelling results. Such an in-depth investigation is beyond the scope of this paper. Nevertheless, our analysis clearly shows that the modification of the incident radiation has a very significant impact on the resulting synthetic spectra. This impact is most prominent in the centre of the Lyman lines - especially the Lyman- $\alpha$ line - and in their integrated intensities. There, a change in the incident radiation can often translate into a large change in these profile characteristics. The impact on the peak intensities of the reversed profiles of Lyman lines is also significant, albeit smaller. Only in the case of the most intense profiles is the impact of the modified illumination on the intensities in the peaks minimal. The change in illumination in the Lyman lines also affects the $\mathrm{H} \alpha$ line profiles to a significant degree despite the fact that the illumination in the $\mathrm{H} \alpha$ line does not change. The $\mathrm{H} \alpha$ is affected because it is formed by the transition from the third to the second level of hydrogen. The populations of these levels are strongly affected by radiation in the Lyman- $\alpha$ and Lyman- $\beta$ lines.

The strong sensitivity of the synthetic spectra to the incident radiation shows that better observations and more detailed studies of the solar disk radiation in the Lyman lines will be needed. This strong sensitivity also means that a detailed forward-modelling analysis of observations of chromospheric and coronal structures will benefit from up-to-date incident radiation boundary conditions. The incident radiation profiles adapted to the date of the analysed observations should be especially considered in a case when the analyzed observations are obtained during maxima of solar activity. This is because the reference incident radiation profiles, both those of Warren et al. (1998) and those provided here, correspond to solar minima. However, careful consideration should be given to the choice of the actual values of the LISIRD composite Lyman- $\alpha$ index or other measures of the variability of solar radiation in Lyman lines.

Acknowledgements. S.G. and P.H. acknowledge support from the grant No. 19 16890S and grant No. 19-17102S of the Czech Science Foundation (GA ČR) S.G. acknowledges support from the grant No. 19-20632S of the Czech Science Foundation (GA ČR). S.G., P.S., P.H., and J.K. acknowledge support from the Joint Mobility Project SAV-18-03 of Academy of Sciences of the Czech Republic and Slovak Academy of Sciences. P.S. and J.K. acknowledge support from the project VEGA 2/0048/20 of the Science Agency. S.G. and P.H. thank for the support from project RVO:67985815 of the Astronomical Institute of the Czech Academy of Sciences. P.S. thanks for the support from the grant No. 1916890 S of the Czech Science Foundation (GA ČR). P.S. and J.K. thank for the support from the grant No. 19-17102S of the Czech Science Foundation (GA ČR). The SUMER project is financially supported by DLR, CNES, NASA, and the ESA PRODEX Programme. SUMER is part of SOHO of ESA and NASA. We acknowledge the use of data obtained from the LASP Interactive Solar Irradiance Data Center (LISIRD) available at lasp.colorado.edu/lisird. The context $\mathrm{H} \alpha$ images used in this paper were obtained by the Big Bear Solar Observatory, which is operated by the New Jersey Institute of Technology, and by the SMART telescope at the Hida Observatory operated by Kyoto University. The authors thank W. Curdt, J.-C. Vial, J. Stěpán and M. Exnerová for valuable discussions.

\section{References}

Allen, C. W. 1976, Astrophysical Quantities (London: The Athlone Press) Artzner, G., Bonnet, R. M., Vial, J. C., et al. 1977, Space Sci. Instrum., 3, 131

Basri, G. S., Linsky, J. L., Bartoe, J. D. F., Brueckner, G., \& van Hoosier, M. E. 1979, ApJ, 230, 924

Bonnet, R. M., Lemaire, P., Vial, J. C., et al. 1978, ApJ, 221, 1032

Bougher, S. W., Roeten, K. J., Olsen, K., et al. 2017, J. Geophys. Res.: Space Phys., 122, 1296

Bzowski, M., Sokół, J. M., Tokumaru, M., et al. 2013, in Solar Parameters for Modeling the Interplanetary Background, eds. E. Quémerais, M. Snow, \& R. M. Bonnet, 13, 67
Combi, M. R., Mäkinen, T. T., Bertaux, J. L., Quémerais, E., \& Ferron, S. 2019, Icarus, 317,610

Curdt, W., Tian, H., Teriaca, L., Schühle, U., \& Lemaire, P. 2008, A\&A, 492, L9 David, K.-H. 1961, ZAp, 53, 37

Dolei, S., Spadaro, D., Ventura, R., et al. 2019, A\&A, 627, A18

Dominique, M., Hochedez, J. F., Schmutz, W., et al. 2013, Sol. Phys., 286, 21 Fontenla, J., Reichmann, E. J., \& Tandberg-Hanssen, E. 1988, ApJ, 329, 464 Gibson, S. E. 2018, Liv. Rev. Sol. Phys., 15, 7

Gladstone, G. R., Stern, S. A., \& Pryor, W. R. 2013, in New Horizons Cruise Observations of Lyman- $\alpha$ Emissions from the Interplanetary Medium, eds. E. Quémerais, M. Snow, \& R. M. Bonnet (New York: Springer Science+Business Media), 13, 177

Gouttebroze, P., Lemaire, P., Vial, J. C., \& Artzner, G. 1978, ApJ, 225, 655

Gunár, S. 2014, in IAU Symposium, eds. B. Schmieder, J. M. Malherbe, \& S. T. Wu, 300, 59

Gunár, S., Heinzel, P., Schmieder, B., Schwartz, P., \& Anzer, U. 2007, A\&A, 472,929

Gunár, S., Heinzel, P., Anzer, U., \& Schmieder, B. 2008, A\&A, 490, 307

Gunár, S., Schwartz, P., Schmieder, B., Heinzel, P., \& Anzer, U. 2010, A\&A, 514, A43

Gunár, S., Mein, P., Schmieder, B., Heinzel, P., \& Mein, N. 2012, A\&A, 543, A93

Gunár, S., Schwartz, P., Dudík, J., et al. 2014, A\&A, 567, A123

Hathaway, D. H. 2015, Liv. Rev. Sol. Phys., 12, 4

Heinzel, P., \& Anzer, U. 2001, A\&A, 375, 1082

Heinzel, P., Gouttebroze, P., \& Vial, J.-C. 1987, A\&A, 183, 351

Heinzel, P., Anzer, U., \& Gunár, S. 2005, A\&A, 442, 331

Heinzel, P., Susino, R., Jejčič, S., Bemporad, A., \& Anzer, U. 2016, A\&A, 589, A128

Kano, R., Trujillo Bueno, J., Winebarger, A., et al. 2017, ApJ, 839, L10

Koutroumpa, D., Quémerais, E., Katushkina, O., et al. 2017, A\&A, 598, A12

Kowalska-Leszczynska, I., Bzowski, M., Sokół, J. M., \& Kubiak, M. A. 2018, ApJ, 852, 115

Kretzschmar, M., Snow, M., \& Curdt, W. 2018, Geophys. Res. Lett., 45, 2138

Leenaarts, J. 2020, Liv. Rev. Sol. Phys., 17, 3

Lemaire, P., Charra, J., Jouchoux, A., et al. 1978, ApJ, 223, L55

Lemaire, P., Emerich, C., Curdt, W., Schuehle, U., \& Wilhelm, K. 1998, A\&A, 334, 1095

Lemaire, P., Emerich, C., Vial, J. C., et al. 2005, Adv. Space Res., 35, 384

Lemaire, P., Vial, J. C., Curdt, W., Schühle, U., \& Woods, T. N. 2012, A\&A, 542, L25

Lemaire, P., Vial, J. C., Curdt, W., Schühle, U., \& Wilhelm, K. 2015, A\&A, 581, A26

Machol, J., Snow, M., Woodraska, D., et al. 2019, Earth Space Sci., 6, 2263

Mein, P. 1991, A\&A, 248, 669

Nina, A., \& Čadež, V. M. 2014, Adv. Space Res., 54, 1276

Pryor, W. R., Snow, M., Quémerais, E., \& Ferron, S. 2013, in Lyman$\alpha$ Observations of Comet Holmes from SORCE SOLSTICE and SOHO SWAN, eds. E. Quémerais, M. Snow, \& R. M. Bonnet (New York: Springer Science+Business Media), 13, 255

Pryor, W. R., Tobiska, W. K., Retherford, K. D., et al. 2020, Lunar and Planetary Science Conference, 1665

Raulin, J.-P., Trottet, G., Kretzschmar, M., et al. 2013, J. Geophys. Res.: Space Phys., 118, 570

Reeves, E. M. 1976, Sol. Phys., 46, 53

Retherford, K. D., Byron, B. D., Magana, L. O., et al. 2020, Lunar and Planetary Science Conference, 2781

Rottman, G. J., \& Woods, T. N. 1994, in Society of Photo-Optical Instrumentation Engineers (SPIE) Conference Series, eds. J. Wang, \& P. B. Hays, Proc. SPIE, 2266, 317

Schühle, U. 2003, SUMER Data Cookbook, http://www2.mps.mpg.de/ projects/soho/sumer/text/cookbook.html

Schühle, U., Brekke, P., Curdt, W., et al. 1998, Appl. Opt., 37, 2646

Schwartz, P., Gunár, S., Jenkins, J. M., et al. 2019, A\&A, 631, A146

Tei, A., Gunár, S., Heinzel, P., et al. 2020, ApJ, 888, 42

Thiemann, E. M. B., Chamberlin, P. C., Eparvier, F. G., et al. 2017, J. Geophys. Res.: Space Phys., 122, 2748

Tian, H., Curdt, W., Marsch, E., \& Schühle, U. 2009a, A\&A, 504, 239

Tian, H., Teriaca, L., Curdt, W., \& Vial, J.-C. 2009b, ApJ, 703, L152

Ueno, S., Nagata, S., Kitai, R., \& Kurokawa, H. 2004, ASP Conf. Ser., 325, 319

Štěpán, J., \& Trujillo Bueno, J. 2013, A\&A, 557, A143

Warren, H. P., Mariska, J. T., \& Wilhelm, K. 1998, ApJS, 119, 105

Wilhelm, K., Curdt, W., Marsch, E., et al. 1995, Sol. Phys., 162, 189

Wilhelm, K., Lemaire, P., Curdt, W., et al. 1997, Sol. Phys., 170, 75

Woods, T. N., Rottman, G., \& Vest, R. 2005, Sol. Phys., 230, 345 


\section{Appendix A: The reference full-disk Lyman- $\alpha$ line profile}

Table A.1. Reference quiet-Sun Lyman- $\alpha$ profile (Fig. 5, left) obtained as an average over eight observed SOHO/SUMER raster scans obtained between June 24, 2008, and June 26, 2008.

\begin{tabular}{|c|c|c|}
\hline Wavelength $\left(\Delta \lambda_{0}\right)[\AA]$ & Intensity $\left[10^{-8} \mathrm{erg} \mathrm{cm}^{-2} \mathrm{~s}^{-1} \mathrm{sr}^{-1} \mathrm{~Hz}^{-1}\right]$ & Intensity $\left[10^{5} \mathrm{erg} \mathrm{cm}^{-2} \mathrm{~s}^{-1} \mathrm{sr}^{-1} \AA^{-1}\right]$ \\
\hline-1.85 & 0.02 & 0.05 \\
\hline-1.81 & 0.03 & 0.05 \\
\hline-1.76 & 0.03 & 0.06 \\
\hline-1.72 & 0.03 & 0.06 \\
\hline-1.68 & 0.03 & 0.06 \\
\hline-1.63 & 0.03 & 0.07 \\
\hline-1.59 & 0.04 & 0.07 \\
\hline-1.55 & 0.04 & 0.08 \\
\hline-1.50 & 0.04 & 0.08 \\
\hline-1.46 & 0.04 & 0.09 \\
\hline-1.42 & 0.05 & 0.09 \\
\hline-1.38 & 0.05 & 0.10 \\
\hline-1.33 & 0.05 & 0.10 \\
\hline-1.29 & 0.05 & 0.11 \\
\hline-1.25 & 0.06 & 0.12 \\
\hline-1.20 & 0.07 & 0.14 \\
\hline-1.16 & 0.08 & 0.15 \\
\hline-1.12 & 0.08 & 0.17 \\
\hline-1.07 & 0.10 & 0.19 \\
\hline-1.03 & 0.11 & 0.22 \\
\hline-0.99 & 0.12 & 0.24 \\
\hline-0.94 & 0.13 & 0.27 \\
\hline-0.90 & 0.15 & 0.30 \\
\hline-0.86 & 0.17 & 0.34 \\
\hline-0.82 & 0.19 & 0.39 \\
\hline-0.77 & 0.22 & 0.45 \\
\hline-0.73 & 0.27 & 0.54 \\
\hline-0.69 & 0.33 & 0.67 \\
\hline-0.64 & 0.41 & 0.84 \\
\hline-0.60 & 0.52 & 1.05 \\
\hline-0.56 & 0.65 & 1.32 \\
\hline-0.51 & 0.84 & 1.70 \\
\hline-0.47 & 1.08 & 2.20 \\
\hline-0.43 & 1.41 & 2.87 \\
\hline-0.39 & 1.86 & 3.78 \\
\hline-0.34 & 2.46 & 5.00 \\
\hline-0.30 & 3.26 & 6.62 \\
\hline-0.26 & 4.14 & 8.42 \\
\hline-0.21 & 4.79 & 9.75 \\
\hline-0.17 & 4.85 & 9.85 \\
\hline-0.13 & 4.24 & 8.61 \\
\hline
\end{tabular}

Notes. The central wavelength $\lambda_{0}$ of the Lyman- $\alpha$ line is $1215.67 \AA$. The uncertainty of the reference profile was estimated to be $\pm(15 / \sqrt{3}) \%$. 
Table A.1. continued.

\begin{tabular}{lcc}
\hline \hline Wavelength $\left(\Delta \lambda_{0}\right)[\AA]$ & Intensity $\left[10^{-8} \mathrm{erg} \mathrm{cm}^{-2} \mathrm{~s}^{-1} \mathrm{sr}^{-1} \mathrm{~Hz}^{-1}\right]$ & Intensity $\left[10^{5} \mathrm{erg} \mathrm{cm}^{-2} \mathrm{~s}^{-1} \mathrm{sr}^{-1} \AA^{-1}\right]$ \\
\hline-0.08 & 3.57 & 7.27 \\
-0.04 & 3.23 & 6.57 \\
0.00 & 3.12 & 6.34 \\
0.04 & 3.19 & 6.48 \\
0.09 & 3.40 & 6.90 \\
0.13 & 3.76 & 7.65 \\
0.17 & 4.27 & 8.68 \\
0.22 & 4.54 & 9.22 \\
0.26 & 4.32 & 8.77 \\
0.30 & 3.70 & 7.51 \\
0.34 & 2.87 & 5.84 \\
0.39 & 2.13 & 4.32 \\
0.43 & 1.57 & 3.19 \\
0.47 & 1.17 & 2.37 \\
0.52 & 0.87 & 1.77 \\
0.56 & 0.67 & 1.36 \\
0.60 & 0.52 & 1.05 \\
0.65 & 0.42 & 0.85 \\
0.69 & 0.35 & 0.72 \\
0.73 & 0.29 & 0.60 \\
0.77 & 0.25 & 0.50 \\
0.82 & 0.20 & 0.41 \\
0.86 & 0.17 & 0.34 \\
0.90 & 0.15 & 0.30 \\
0.95 & 0.13 & 0.27 \\
0.99 & 0.12 & 0.24 \\
1.03 & 0.11 & 0.22 \\
1.08 & 0.10 & 0.20 \\
1.12 & 0.09 & 0.18 \\
1.16 & 0.08 & 0.17 \\
1.20 & 0.08 & 0.15 \\
1.25 & 0.07 & 0.14 \\
1.29 & 0.06 & 0.13 \\
1.33 & 0.06 & 0.12 \\
1.38 & 0.06 & 0.11 \\
1.42 & 0.05 & 0.11 \\
1.46 & 0.05 & 0.10 \\
1.51 & 0.05 & 0.10 \\
1.55 & 0.05 & 0.09 \\
1.59 & 0.04 & 0.09 \\
1.64 & 0.04 & 0.08 \\
1.68 & 0.04 & 0.08 \\
1.72 & 0.04 & 0.08 \\
1.76 & 0.04 & 0.07 \\
1.81 & 0.03 & 0.07 \\
1.85 & 0.03 & 0.07 \\
\hline
\end{tabular}


Table A.2. Symmetrized reference Lyman- $\alpha$ profile (Fig. 5, right).

\begin{tabular}{lcc}
\hline \hline Wavelength $\left(\Delta \lambda_{0}\right)[\AA]$ & Intensity $\left[10^{-8} \mathrm{erg} \mathrm{cm}^{-2} \mathrm{~s}^{-1} \mathrm{sr}^{-1} \mathrm{~Hz}^{-1}\right]$ & Intensity $\left[10^{5} \mathrm{erg} \mathrm{cm}^{-2} \mathrm{~s}^{-1} \mathrm{sr}^{-1} \AA^{-1}\right]$ \\
\hline 0.00 & 3.12 & 6.33 \\
0.02 & 3.16 & 6.42 \\
0.04 & 3.21 & 6.51 \\
0.06 & 3.33 & 6.76 \\
0.08 & 3.46 & 7.02 \\
0.10 & 3.67 & 7.45 \\
0.12 & 3.91 & 7.94 \\
0.14 & 4.16 & 8.44 \\
0.16 & 4.42 & 8.97 \\
0.18 & 4.58 & 9.29 \\
0.20 & 4.63 & 9.40 \\
0.22 & 4.60 & 9.34 \\
0.24 & 4.40 & 8.93 \\
0.26 & 4.18 & 8.48 \\
0.28 & 3.83 & 7.77 \\
0.31 & 3.29 & 6.69 \\
0.34 & 2.73 & 5.54 \\
0.38 & 2.09 & 4.25 \\
0.42 & 1.60 & 3.25 \\
0.47 & 1.15 & 2.33 \\
0.52 & 0.83 & 1.69 \\
0.57 & 0.62 & 1.26 \\
0.62 & 0.47 & 0.96 \\
0.68 & 0.35 & 0.72 \\
0.78 & 0.23 & 0.47 \\
0.88 & 0.16 & 0.32 \\
0.98 & 0.12 & 0.25 \\
1.08 & 0.10 & 0.20 \\
1.18 & 0.08 & 0.15 \\
1.30 & 0.06 & 0.12 \\
1.50 & 0.04 & 0.09 \\
\hline
\end{tabular}

Notes. The central wavelength $\lambda_{0}$ of the Lyman- $\alpha$ line is $1215.67 \AA$. The uncertainty of the reference profile was estimated to be $\pm(15 / \sqrt{3}) \%$. 
S. Gunár et al.: Quiet-Sun hydrogen Lyman- $\alpha$ line profile

Appendix B: Coefficients describing the variation of solar radiation in Lyman lines

Table B.1. Coefficients describing the variation of the solar radiation in the Lyman- $\alpha$ and higher Lyman lines.

\begin{tabular}{|c|c|c|c|}
\hline Date & $\begin{array}{l}\text { Value of 400-days averaged } \\
\text { LISIRD index }\left[10^{-2} \mathrm{~W} \mathrm{~m}^{-2}\right]\end{array}$ & $\begin{array}{l}\text { Coefficient for } \\
\text { Lyman- } \alpha \text { line }\end{array}$ & $\begin{array}{c}\text { Coefficient for } \\
\text { higher Lyman lines }\end{array}$ \\
\hline 1995/01/01 & 0.644 & 1.065 & 1.037 \\
\hline $1995 / 05 / 01$ & 0.642 & 1.062 & 1.033 \\
\hline $1995 / 09 / 01$ & 0.635 & 1.050 & 1.022 \\
\hline 1996/01/01 & 0.627 & 1.038 & 1.010 \\
\hline $1996 / 05 / 01$ & 0.622 & 1.028 & 1.001 \\
\hline $1996 / 05 / 15$ & 0.621 & 1.027 & 1.000 \\
\hline 1996/09/01 & 0.620 & 1.026 & 0.998 \\
\hline $1997 / 01 / 01$ & 0.622 & 1.029 & 1.001 \\
\hline $1997 / 05 / 01$ & 0.634 & 1.048 & 1.020 \\
\hline $1997 / 09 / 01$ & 0.654 & 1.081 & 1.052 \\
\hline 1998/01/01 & 0.684 & 1.131 & 1.101 \\
\hline $1998 / 05 / 01$ & 0.725 & 1.199 & 1.168 \\
\hline 1998/09/01 & 0.765 & 1.266 & 1.232 \\
\hline $1999 / 01 / 01$ & 0.798 & 1.321 & 1.286 \\
\hline 1999/05/01 & 0.822 & 1.360 & 1.324 \\
\hline $1999 / 09 / 01$ & 0.847 & 1.400 & 1.363 \\
\hline $2000 / 01 / 01$ & 0.876 & 1.449 & 1.410 \\
\hline $2000 / 05 / 01$ & 0.895 & 1.479 & 1.440 \\
\hline $2000 / 09 / 01$ & 0.894 & 1.478 & 1.439 \\
\hline 2001/01/01 & 0.879 & 1.455 & 1.416 \\
\hline $2001 / 05 / 01$ & 0.874 & 1.445 & 1.407 \\
\hline $2001 / 09 / 01$ & 0.894 & 1.478 & 1.439 \\
\hline $2002 / 01 / 01$ & 0.905 & 1.498 & 1.458 \\
\hline $2002 / 05 / 01$ & 0.908 & 1.502 & 1.463 \\
\hline 2002/09/01 & 0.864 & 1.429 & 1.392 \\
\hline $2003 / 01 / 01$ & 0.823 & 1.361 & 1.325 \\
\hline $2003 / 05 / 01$ & 0.797 & 1.318 & 1.283 \\
\hline $2003 / 09 / 01$ & 0.768 & 1.271 & 1.237 \\
\hline 2004/01/01 & 0.755 & 1.248 & 1.215 \\
\hline $2004 / 05 / 01$ & 0.734 & 1.214 & 1.182 \\
\hline $2004 / 09 / 01$ & 0.715 & 1.183 & 1.151 \\
\hline $2005 / 01 / 01$ & 0.702 & 1.161 & 1.130 \\
\hline $2005 / 05 / 01$ & 0.687 & 1.137 & 1.107 \\
\hline $2005 / 09 / 01$ & 0.668 & 1.105 & 1.076 \\
\hline $2006 / 01 / 01$ & 0.657 & 1.087 & 1.058 \\
\hline $2006 / 05 / 01$ & 0.648 & 1.072 & 1.043 \\
\hline $2006 / 09 / 01$ & 0.643 & 1.063 & 1.035 \\
\hline $2007 / 01 / 01$ & 0.636 & 1.052 & 1.024 \\
\hline $2007 / 05 / 01$ & 0.626 & 1.034 & 1.007 \\
\hline
\end{tabular}

Notes. The coefficients were derived from a 400-days averaged LISIRD Lyman- $\alpha$ composite index (see Sect. 3.1) for selected dates throughout the lifetime of SOHO. Coefficients for the Lyman- $\alpha$ line are computed with respect to the date of observations used in the present work (June 25, 2008). Coefficients for the higher Lyman lines are computed with respect to the date of observations used in Warren et al. (1998), i.e. May 15, 1996. 
Table B.1. continued.

\begin{tabular}{|c|c|c|c|}
\hline Date & $\begin{array}{l}\text { Value of 400-days averaged } \\
\text { LISIRD index }\left[10^{-2} \mathrm{~W} \mathrm{~m}^{-2}\right]\end{array}$ & $\begin{array}{l}\text { Coefficient for } \\
\text { Lyman- } \alpha \text { line }\end{array}$ & $\begin{array}{c}\text { Coefficient for } \\
\text { higher Lyman lines }\end{array}$ \\
\hline $2007 / 09 / 01$ & 0.615 & 1.018 & 0.991 \\
\hline $2008 / 01 / 01$ & 0.609 & 1.008 & 0.981 \\
\hline $2008 / 05 / 01$ & 0.605 & 1.001 & 0.974 \\
\hline $2008 / 06 / 25$ & 0.605 & 1.000 & 0.973 \\
\hline $2008 / 09 / 01$ & 0.603 & 0.997 & 0.970 \\
\hline $2009 / 01 / 01$ & 0.602 & 0.996 & 0.970 \\
\hline $2009 / 05 / 01$ & 0.604 & 0.999 & 0.973 \\
\hline $2009 / 09 / 01$ & 0.613 & 1.014 & 0.987 \\
\hline $2010 / 01 / 01$ & 0.625 & 1.033 & 1.006 \\
\hline $2010 / 05 / 01$ & 0.640 & 1.059 & 1.031 \\
\hline $2010 / 09 / 01$ & 0.653 & 1.080 & 1.051 \\
\hline $2011 / 01 / 01$ & 0.674 & 1.115 & 1.086 \\
\hline $2011 / 05 / 01$ & 0.704 & 1.164 & 1.133 \\
\hline $2011 / 09 / 01$ & 0.738 & 1.221 & 1.189 \\
\hline $2012 / 01 / 01$ & 0.752 & 1.243 & 1.210 \\
\hline $2012 / 05 / 01$ & 0.761 & 1.258 & 1.225 \\
\hline $2012 / 09 / 01$ & 0.749 & 1.238 & 1.205 \\
\hline $2013 / 01 / 01$ & 0.753 & 1.245 & 1.212 \\
\hline $2013 / 05 / 01$ & 0.757 & 1.253 & 1.220 \\
\hline $2013 / 09 / 01$ & 0.777 & 1.285 & 1.251 \\
\hline $2014 / 01 / 01$ & 0.793 & 1.311 & 1.276 \\
\hline $2014 / 05 / 01$ & 0.806 & 1.333 & 1.298 \\
\hline $2014 / 09 / 01$ & 0.813 & 1.344 & 1.308 \\
\hline $2015 / 01 / 01$ & 0.808 & 1.337 & 1.301 \\
\hline $2015 / 05 / 01$ & 0.791 & 1.308 & 1.274 \\
\hline $2015 / 09 / 01$ & 0.762 & 1.261 & 1.227 \\
\hline $2016 / 01 / 01$ & 0.727 & 1.202 & 1.170 \\
\hline $2016 / 05 / 01$ & 0.703 & 1.162 & 1.131 \\
\hline $2016 / 09 / 01$ & 0.671 & 1.109 & 1.080 \\
\hline $2017 / 01 / 01$ & 0.651 & 1.076 & 1.047 \\
\hline $2017 / 05 / 01$ & 0.637 & 1.054 & 1.026 \\
\hline $2017 / 09 / 01$ & 0.629 & 1.040 & 1.012 \\
\hline $2018 / 01 / 01$ & 0.621 & 1.028 & 1.001 \\
\hline $2018 / 05 / 01$ & 0.612 & 1.013 & 0.986 \\
\hline $2018 / 09 / 01$ & 0.606 & 1.002 & 0.975 \\
\hline $2019 / 01 / 01$ & 0.604 & 0.999 & 0.972 \\
\hline $2019 / 05 / 01$ & 0.599 & 0.991 & 0.965 \\
\hline $2019 / 09 / 01$ & 0.599 & 0.991 & 0.965 \\
\hline $2020 / 01 / 01$ & 0.599 & 0.990 & 0.964 \\
\hline $2020 / 05 / 01$ & 0.601 & 0.993 & 0.967 \\
\hline
\end{tabular}

